Draft VERSION June 26, 2018

Preprint typeset using LATEX style AASTeX6 v. 1.0

\title{
QUANTIFYING THE INTERSTELLAR MEDIUM AND COSMIC RAYS IN THE MBM 53, 54, AND 55 MOLECULAR CLOUDS AND THE PEGASUS LOOP USING FERMI-LAT GAMMA-RAY OBSERVATIONS
}

\author{
T. Mizuno ${ }^{1}$, S. Abdollahi ${ }^{2}$, Y. Fukui ${ }^{3}$, K. Hayashi ${ }^{3}$, A. Okumura $^{4}$, H. Tajima ${ }^{4,5}$, and H. Yamamoto ${ }^{3}$ \\ ${ }^{1}$ Hiroshima Astrophysical Science Center, Hiroshima University, Higashi-Hiroshima, Hiroshima 739-8526, Japan; \\ mizuno@hep01.hepl.hiroshima-u.ac.jp \\ ${ }^{2}$ Department of Physical Sciences, Hiroshima University, Higashi-Hiroshima, Hiroshima 739-8526, Japan \\ ${ }^{3}$ Department of Physics and Astrophysics, Nagoya University, Chikusa-ku Nagoya 464-8602, Japan \\ ${ }^{4}$ Nagoya University, Institute for Space-Earth Environmental Research, Furo-cho, Chikusa-ku, Nagoya 464-8601, Japan \\ ${ }^{5}$ W. W. Hansen Experimental Physics Laboratory, Kavli Institute for Particle Astrophysics and Cosmology, Department of Physics and \\ SLAC National Accelerator Laboratory, Stanford University, Stanford, CA 94305, USA
}

\begin{abstract}
A study of the interstellar medium (ISM) and cosmic rays (CRs) using Fermi Large Area Telescope (LAT) data, in a region encompassing the nearby molecular clouds MBM 53, 54, and 55 and a farinfrared loop-like structure in Pegasus, is reported. By comparing Planck dust thermal emission model with Fermi-LAT $\gamma$-ray data, it was found that neither the dust radiance $(R)$ nor the dust opacity at $353 \mathrm{GHz}\left(\tau_{353}\right)$ were proportional to the total gas column density $N\left(\mathrm{H}_{\text {tot }}\right)$ primarily because $N\left(\mathrm{H}_{\text {tot }}\right) / R$ and $N\left(\mathrm{H}_{\text {tot }}\right) / \tau_{353}$ depend on the dust temperature $\left(T_{\mathrm{d}}\right)$. The $N\left(\mathrm{H}_{\text {tot }}\right)$ distribution was evaluated using $\gamma$-ray data by assuming the regions of high $T_{\mathrm{d}}$ to be dominated by optically thin atomic hydrogen (HI) and by employing an empirical linear relation of $N\left(\mathrm{H}_{\mathrm{tot}}\right) / R$ to $T_{\mathrm{d}}$. It was determined that the mass of the gas not traced by the 21 -cm or $2.6-\mathrm{mm}$ surveys is $\sim 25 \%$ of the mass of $\mathrm{HI}$ in the optically thin case and is larger than the mass of the molecular gas traced by carbon monoxide by a factor of up to 5 . The measured $\gamma$-ray emissivity spectrum is consistent with a model based on CR spectra measured at the Earth and the nuclear enhancement factor of $\leq 1.5$. It is, however, lower than local H I emissivities reported by previous Fermi-LAT studies employing different analysis methods and assumptions on ISM properties by $15 \%-20 \%$ in energies below a few $\mathrm{GeV}$, even if we take account of the statistical and systematic uncertainties. The origin of the discrepancy is also discussed.
\end{abstract}

Keywords: ISM: general — cosmic rays — gamma rays: ISM

\section{INTRODUCTION}

Interstellar space is permeated with ordinary matter (gas or dust), which is known as the interstellar medium (ISM), high-energy charged particles known as cosmic rays (CRs), interstellar radiation fields (ISRF), and magnetic fields. These constituents have comparable pressures and are mutually interacting. They play an important role in many physical and chemical processes (e.g., star formation) that occur in the Milky Way and have been studied in various wavebands - from radio to X-rays to $\gamma$ rays (for a review, see, e.g., Ferriere 2001). Of the multiwavelength observations, cosmic $\gamma$-ray emission is known to be a powerful probe to study the ISM and Galactic CRs. High-energy CR protons and electrons interact with the interstellar gas or the ISRF and produce $\gamma$ rays through nucleon-nucleon interactions, electron bremsstrahlung, and inverse Compton (IC) scattering. Because the ISM is essentially transparent to these high-energy photons, we can study the ISM distribution via $\gamma$-ray observations. Because the $\gamma$-ray production cross section is independent of the chemical or thermodynamic state of the interstellar gas, cosmic $\gamma$ rays have been recognized as a unique tracer of the total gas column density regardless of its atomic or molecular state. If the gas column densities are estimated with good accuracy using observations in other wavebands such as radio, infrared, and optical, the CR spectrum and density distribution can be examined as well. In fact, the distributions of ISM and CRs obtained are ambiguous because of the degeneracy; therefore, $\gamma$-ray observations need to be complemented by using data from other wavebands.

Usually, the distribution of atomic hydrogen (HI) is measured by 21-cm line surveys (e.g., Dickey \& Lockman 1990), and the distribution of molecular hydrogen $\left(\mathrm{H}_{2}\right)$ is derived via 2.6- $\mathrm{mm}$ line observations of carbon monoxide, $\mathrm{CO}$ (e.g., 
Dame et al. 2001). The total gas column density can also be estimated from extinction, reddening, or emission by dust (e.g., Bohlin et al. 1978). These tracers have advantages and disadvantages. H I 21-cm line surveys directly trace the distribution of atomic hydrogen and provide us with the line velocity along the line of sight inferred from the Doppler shift, which, in turn, provides distance information under the assumption of the Galactic rotation curve (e.g., Clemens 1985). The obtained H I column density, however, suffers from uncertainties of the 21-cm line opacity and self absorption. CO 2.6-mm line surveys also provide us with velocity (and distance) information, although it is an indirect tracer of $\mathrm{H}_{2}$ and the derived molecular gas column density is affected by the assumption of the conversion factor (the so-called $X_{\mathrm{CO}}$ ). This method may also miss CO-dark $\mathrm{H}_{2}$ clouds due to, e.g., photodissociation (Wolfire et al. 2010). Dust is expected to be well mixed with gas in the cold and warm phases of the ISM and is a probe of the total gas column density, although it lacks velocity (and distance) information. Because dust is an indirect tracer of the interstellar gas (like $\mathrm{CO}$ is a tracer of $\mathrm{H}_{2}$ ), the derived gas column density is affected by assumptions of the dust-to-gas ratio and dust emissivity (or extinction). Therefore, comparing $\mathrm{H}$, CO, and dust observations is crucial to study the interstellar gas distribution, and adding $\gamma$-ray data is important because it is another independent tracer of the total gas column density.

Studies of the ISM (and CRs) have advanced significantly in last two decades. The G236+39 cloud was found to have significant infrared emission from dust, which was not accounted for by the H I 21-cm or CO 2.6-mm line observations, suggesting the presence of an $\mathrm{H}_{2}$ cloud with $\mathrm{CO}$ emission below the detection threshold (Reach et al. 1994). Combining the EGRET $\gamma$-ray data, H I, CO, and dust extinction maps, significant amount of gas not traced by the H I or CO surveys was revealed in the solar neighborhood and has been referred to as "dark gas" (Grenier et al. 2005). This work has been confirmed and improved in terms of significance and accuracy by recent observations by Fermi-LAT (e.g., Abdo et al. 2010; Ackermann et al. 2011, 2012b). Taking account of the dark gas also makes it possible to obtain information on Galactic CRs with unprecedented accuracy (e.g., Casandjian 2015). The Planck satellite provides an accurate dust thermal emission model, which is crucial to study the ISM. By comparing the Planck dust emission model, and the H I and CO data, the Planck Collaboration (2011) estimated the mass of dark gas to be $\sim 30 \%$ of the atomic gas and $\sim 120 \%$ of the CO-bright molecular gas in the solar neighborhood. By comparing the Planck dust optical depth map at $353 \mathrm{GHz}\left(\tau_{353}\right)$, and the $\mathrm{HI} / \mathrm{CO}$ data and assuming that the total gas column density was proportional to $\tau_{353}$, Fukui et al. (2014, 2015) proposed that a significant amount of the atomic hydrogen was optically thick in areas with low dust temperature $\left(T_{\mathrm{d}}\right)$, resulting in an excess mass comparable to the mass of H I in the optically thin case. The Planck Collaboration (2014), on the other hand, found that the dust radiance $R$ (bolometric luminosity) was well correlated with the integrated $\mathrm{HI} 21-\mathrm{cm}$ line intensity, $W_{\mathrm{H}}$, in wide range of $T_{\mathrm{d}}$ in the diffuse ISM, and proposed that it would be a better tracer of the dust (and the total gas) column density. The Planck Collaboration (2015) combined the Fermi-LAT data and Planck dust emission model to study the ISM in the Chamaeleon molecular cloud. They employed a detailed model of the dust emission by Draine \& Li (2007) and found a good correlation with $\gamma$-ray data. The obtained mass of the dark gas was approximately twice that of the CO-bright $\mathrm{H}_{2}$ and contributed $\sim 15 \%$ of the total gas mass.

Here, we report an analysis of the Fermi-LAT $\gamma$-ray data in the Galactic longitudes $60^{\circ} \leq l \leq 120^{\circ}$ and the Galactic latitudes $-60^{\circ} \leq b \leq-28^{\circ}$. Our region of interest (ROI) encompasses the MBM 53, 54, and 55 molecular cloud complexes (located at $l=84^{\circ}$ to $96^{\circ}$ and $b=-44^{\circ}$ to $-30^{\circ}$ ) and an infrared loop-like structure in Pegasus (area of $\sim 20^{\circ} \times 20^{\circ}$ around $\left.(l, b) \sim\left(109^{\circ},-45^{\circ}\right)\right)$. MBM 53, 54, and 55 are some of the nearest large molecular clouds (Yamamoto et al. 2003), located at a distance of $\sim 150$ pc estimated by Welty et al. (1989) based on measurements of interstellar NaI absorption toward stars associated with the clouds. The loop-like structure in Pegasus (hereafter termed the "Pegasus loop") was identified in IRAS $100 \mu \mathrm{m}$ maps (Kiss et al. 2004) and studied in CO using the NANTEN telescope (Yamamoto et al. 2006); its distance has been estimated to be $\sim 100$ pc which is equal to the distance of the B2 star in the center of the loop. The MBM 53, 54, and 55 clouds and the Pegasus loop are nearby (100-150 pc) molecular clouds located at high Galactic latitudes (having small overlap with structures in the ISM at different distances), and therefore are expected to have uniform ISM and CR properties (e.g., dust-to-gas ratio and CR density).

This paper is organized as follows. We describe the properties of the ISM tracers in the complexes studied in Section 2 , and the $\gamma$-ray observations, data selection, and modeling in Section 3 . The results of the data analysis are presented in Section 4, where we find that neither $R$ nor $\tau_{353}$ are good measures of the total gas column density $\left[N\left(\mathrm{H}_{\text {tot }}\right)\right]$. We use the Fermi-LAT $\gamma$-ray data to compensate for the observed $T_{\mathrm{d}}$ dependence and evaluate $N\left(\mathrm{H}_{\text {tot }}\right)$ (also shown in Section 4). We discuss the ISM and CR properties of the studied region in Section 5. A summary of this study and future prospects are presented in Section 6 .

Before describing the analysis and results of the study, we note the difference of our approach from that of preceding 
studies. Most previous Fermi-LAT studies of diffuse $\gamma$-ray emission used H I, CO, and dust data to prepare template maps of the neutral gas distribution in the atomic phase, the molecular phase, and the dark gas phase, respectively, and analyzed $\gamma$-ray data to study the ISM and CRs (the method is hereafter called a "conventional template-fitting method"). Motivated by our finding that the ratio of the $\gamma$-ray intensity associated with the ISM gas [i.e., a tracer of $N\left(\mathrm{H}_{\text {tot }}\right)$ ] to dust tracers $\left(R\right.$ or $\left.\tau_{353}\right)$ depends on $T_{\mathrm{d}}$, we took a different approach: we focus on evaluating $N\left(\mathrm{H}_{\text {tot }}\right)$ using the Planck dust map by applying the correction based on $T_{\mathrm{d}}$ in the $\gamma$-ray data analysis (Section 4.3), and then discuss the relation of the obtained $N\left(\mathrm{H}_{\text {tot }}\right)$ distribution with $\mathrm{HI} 21-\mathrm{cm}$ and $\mathrm{CO} 2.6-\mathrm{mm}$ line intensities (Section 5). A comparison with a conventional template-fitting method is also given in Section 5 and Appendix D. 


\section{PROPERTIES OF THE ISM TRACERS}

We analyzed the $\gamma$-ray data in a region with Galactic longitude $60^{\circ} \leq l \leq 120^{\circ}$ and Galactic latitude $-60^{\circ} \leq$ $b \leq-28^{\circ}$, which encompasses the MBM 53, 54, and 55 cloud complexes and the Pegasus loop. Because preparing good templates of the interstellar gas is crucial for $\gamma$-ray data analysis, we first investigated the properties of the ISM tracers. We prepared dust maps, a $W_{\mathrm{H} \text { I }}$ map, and an integrated CO 2.6-mm line intensity ( $\left.W_{\mathrm{CO}}\right)$ map, all stored in a HEALPix (Górski et al. 2005) equal-area sky map of order 9 (pixel size is $\sim 0.013 \operatorname{deg}^{2}$ ). We used the Planck dust maps (of $R, \tau_{353}, T_{\mathrm{d}}$, and dust spectral index $\beta$ ) of the public data release 1 (the version $\left.\mathrm{R} 1.20\right)^{1}$ described by Planck Collaboration (2014), since the latest release (public data release 2) does not include the dust radiance map. Assuming a uniform dust temperature along the line of sight, they have modeled the dust thermal emission with a single modified black-body, and constructed those maps (for details of the procedure, see Planck Collaboration 2014). As described in Planck Collaboration (2014), the dust optical depth is the product of the dust opacity (cross section) per $\mathrm{H}$ atom and the total gas column density. Therefore if the dust cross section is uniform $\tau_{353}$ is proportional to $N\left(\mathrm{H}_{\text {tot }}\right)$. The dust radiance $R$ is also expected to trace the total gas column density, since it is proportional to $N\left(\mathrm{H}_{\text {tot }}\right)$ under the assumption of a uniform dust-to-gas ratio, dust emissivity, and ISRF (see also Section 4.2).

To construct the $W_{\mathrm{H} \text { I }}$ map, we referred to the Leiden/Argentine/Bonn (LAB) survey (Kalberla et al. 2005) integrated over the velocity range from -450 to $400 \mathrm{~km} \mathrm{~s}^{-1} .{ }^{2}$ We used a $W_{\mathrm{CO}}$ map internally available to the LAT team, which combines the work by Dame et al. (2001) and new data at high Galactic latitudes sampled in 0.25. The new CO data includes most of the high-latitude CO clouds in the region studied here. The CO spectra were filtered to suppress the noise and integrated over velocities (Dame 2011). We converted the $W_{\mathrm{H} \text { I }}$ map into the column density $N(\mathrm{HI})$ using the optically thin approximation $\left[N\left(\mathrm{HI}_{\mathrm{thin}}\right)\left(\mathrm{cm}^{-2}\right)=1.82 \times 10^{18} \cdot W_{\mathrm{H}}\left(\mathrm{K} \mathrm{km} \mathrm{s}^{-1}\right)\right]$. The obtained $N(\mathrm{HI})$ model map, the $\mathrm{W}_{\mathrm{CO}}$ map $\left(\mathrm{K} \mathrm{km} \mathrm{s}^{-1}\right)$, and the $T_{\mathrm{d}}$ map $(\mathrm{K})$ in our ROI are shown in Figure 1 . In the Planck dust maps, we identified several areas with high $T_{\mathrm{d}}$ indicating localized heating by stars. We refilled these areas (in the $R$, $\tau_{353}$, and $T_{\mathrm{d}}$ maps), with the average of the peripheral pixels. Details of this procedure are described in Appendix A.

The correlations between $W_{\mathrm{H} \text { I }}$ and $R$, and those between $W_{\mathrm{H}_{\mathrm{I}}}$ and $\tau_{353}$, are shown in Figure 2 , in which the colors represent different dust temperatures. We masked areas with $W_{\mathrm{CO}}$ intensity greater than $1.1 \mathrm{~K} \mathrm{~km} \mathrm{~s}$ in order to match the procedure of Fukui et al. (2014), who analyzed the ISM in and around the MBM 53, 54, and 55 clouds. Therefore the regions of $\mathrm{H}_{2}$ associated with appreciable $W_{\mathrm{CO}}$ are not included in the figure. We can confirm the trends of the dust-gas relation found by previous studies described in Section 1 as (1) we observe in Figure 2a a good correlation between the $W_{\mathrm{H}_{\mathrm{I}}}$ and $R$ in a wide range of $T_{\mathrm{d}}$ (Planck Collaboration 2014) ${ }^{3}$ and (2) we observe in Figure $2 \mathrm{~b}$ a strong $T_{\mathrm{d}}$ dependence of the $W_{\mathrm{H}}-\tau_{353}$ relation, which Fukui et al. (2014) interpreted to be primarily due to optically thick $\mathrm{H}$ in low- $T_{\mathrm{d}}$ areas.

Although the region studied is dominated by the local ISM, contamination from clouds with different velocities (and therefore likely having different distances) is inevitable. We identified ISM clouds with velocities in -80 to $-30 \mathrm{~km} \mathrm{~s}^{-1}$ [reported by Wakker (2001) as some of intermediate-velocity clouds (IVCs) in the southern sky], while the main clouds have velocities in -30 to $+20 \mathrm{~km} \mathrm{~s}^{-1}$ (see Appendix B for details). We masked the areas shown in Figure B1b to eliminate the contribution from the IVCs and confirmed the same trends as described above. We also examined the dust- $W_{\mathrm{H} \text { I }}$ relation in sub-regions: one is in $80^{\circ} \leq l \leq 100^{\circ}$ and $-44^{\circ} \leq b \leq-28^{\circ}$ which covers the MBM 53, 54, and 55 clouds, and the other is in $100^{\circ} \leq l \leq 120^{\circ}$ and $-55^{\circ} \leq b \leq-35^{\circ}$ which covers the Pegasus loop. Again, we confirmed the same trends as described above; the difference seen among sub-regions is smaller than the difference seen between two tracers $\left(R\right.$ and $\left.\tau_{353}\right)$.

The correlation between the dust tracers and $W_{\mathrm{H} \text { I }}$ alone is not sufficient to distinguish which $\left(R\right.$ or $\left.\tau_{353}\right)$ is the better tracer of the total dust (and gas) column density. We therefore prepared two types of $N\left(\mathrm{H}_{\text {tot }}\right)$ model maps based on $R$ and $\tau_{353}$ and tested them against the Fermi-LAT $\gamma$-ray data. We started with a single $N\left(\mathrm{H}_{\text {tot }}\right)$ map $(\mathrm{Section} 4.1)$ and then employed multiple $N\left(\mathrm{H}_{\text {tot }}\right)$ maps sorted by $T_{\mathrm{d}}$ (Section 4.2$)$. We finally came back to a single $N\left(\mathrm{H}_{\text {tot }}\right)$ map with a $T_{\mathrm{d}}$-dependent correction applied in order to better represent the $\gamma$-ray data (Section 4.3 ).

\footnotetext{
1 http://irsa.ipac.caltech.edu/data/Planck/release_1/all-sky-maps/

2 According to Kalberla et al. (2005), the velocity range of the survey spanned $850 \mathrm{~km} \mathrm{~s}^{-1}$ at a resolution of $1.3 \mathrm{~km} \mathrm{~s}{ }^{-1}$ with a rootmean-square (RMS) noise per channel of 0.07-0.09 K. Therefore, the RMS noise in the integrated intensity over the entire velocity range is estimated to be $\sim 0.08 \mathrm{~K} \times \sqrt{850 / 1.3} \times 1.3 \mathrm{~km} \mathrm{~s}^{-1} \sim 2.7 \mathrm{~K} \mathrm{~km} \mathrm{~s}^{-1}$, much smaller than the values of $W_{\mathrm{H} \text { I }}$ in our ROI.

3 They reported a good correlation up to column densities of (at least) $5 \times 10^{20} \mathrm{~cm}^{-2}$ (Figure 20 of the reference), which corresponds to $W_{\mathrm{H} \text { I }}$ of $\sim 280 \mathrm{~K} \mathrm{~km} \mathrm{~s}^{-1}$.
} 


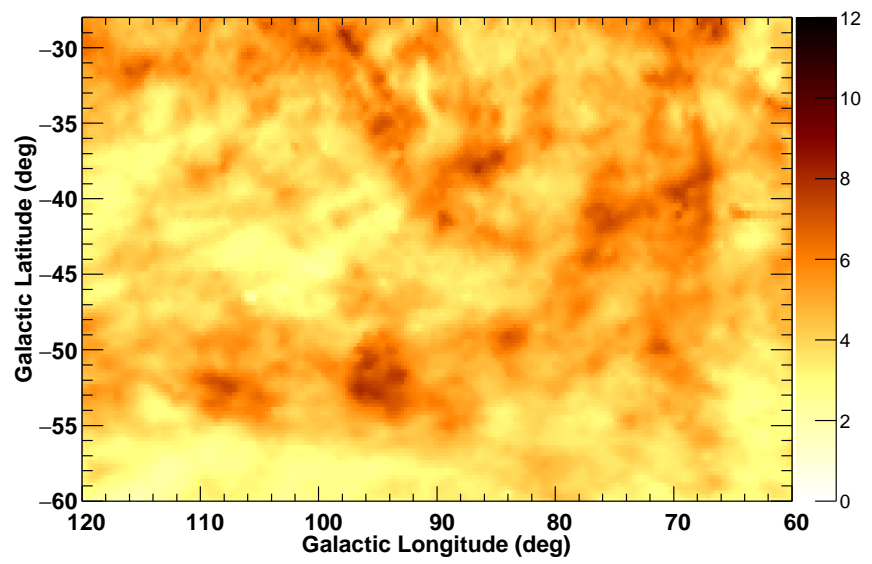

(a)

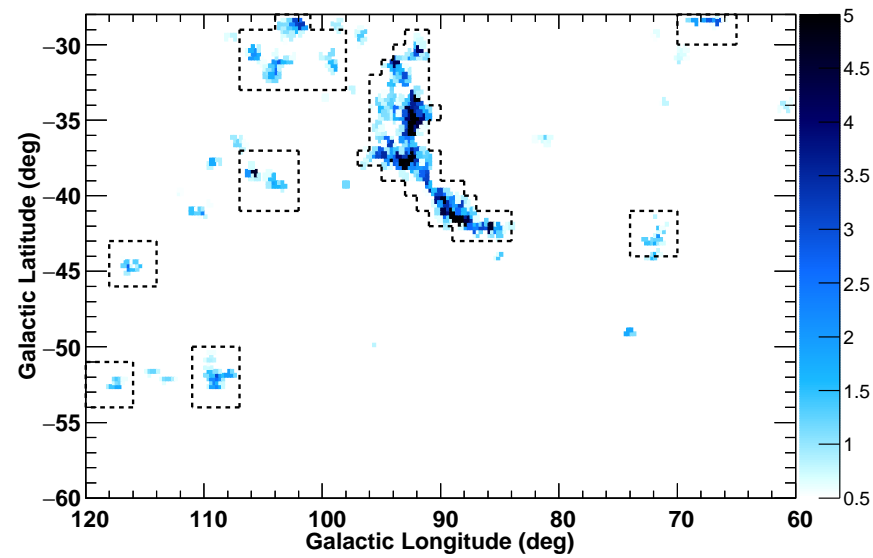

(b)

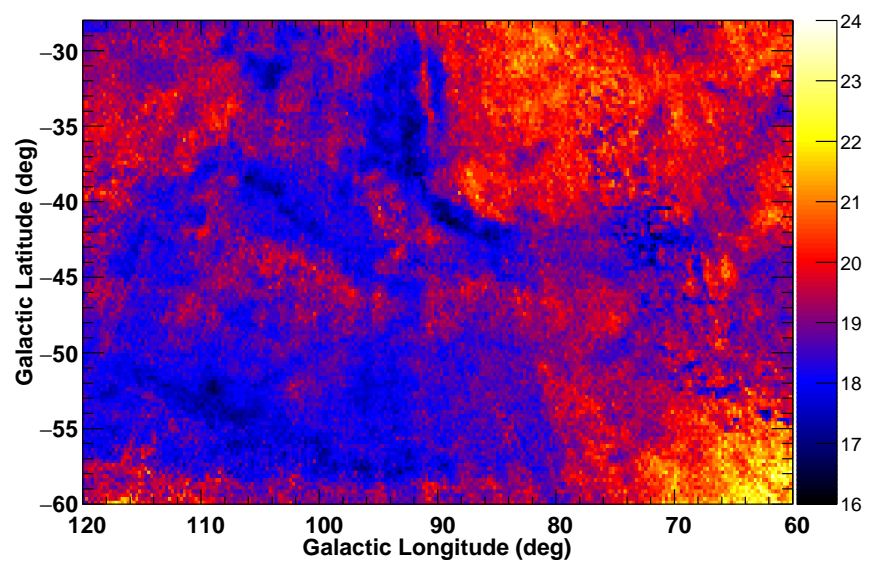

(c)

Figure 1. (a) The $W_{\mathrm{H} \text { I }}$ map converted into $N(\mathrm{HI})$ with the optically-thin approximation $\left(N\left(\mathrm{H} \mathrm{I}_{\mathrm{thin}}\right)\left(\mathrm{cm}^{-2}\right)=1.82 \times 10^{18}\right.$. $\left.W_{\mathrm{HI}}\left(\mathrm{K} \mathrm{km} \mathrm{s}^{-1}\right)\right)$, shown in units of $10^{20} \mathrm{~cm}^{-2}$; (b) the $\mathrm{W}_{\mathrm{CO}} \operatorname{map}\left(\mathrm{K} \mathrm{km} \mathrm{s}^{-1}\right)$; and (c) the $T_{\mathrm{d}}$ map $(\mathrm{K})$. The dotted lines in panel (b) indicate the areas to be masked in Section 4.2. 


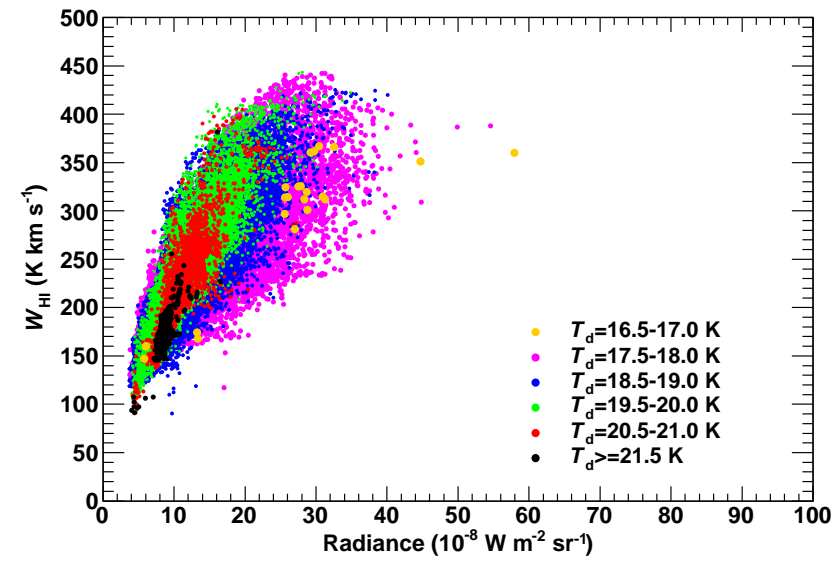

(a)

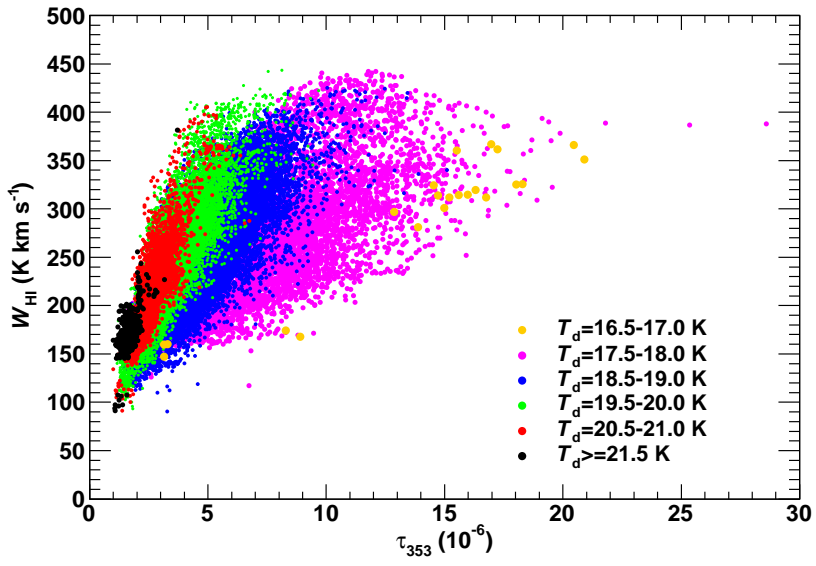

(b)

Figure 2. The correlations between $W_{\mathrm{H} \text { I }}$ and dust tracers: (a) scatter plot of $W_{\mathrm{H} \text { I }}$ versus $R$ and (b) scatter plot of $W_{\mathrm{H} \text { I }}$ versus $\tau_{353}$. Data are shown in $0.5 \mathrm{~K}$ ranges of $T_{\mathrm{d}}$ with $0.5 \mathrm{~K}$ gaps between intervals for clarity. Each point represents each pixel of our HEALPix map (order 9; pixel size is $\sim 0.013 \mathrm{deg}^{2}$ ). 


\section{GAMMA-RAY DATA AND MODELING}

\subsection{Gamma-ray Observations and Data Selection}

The LAT on board the Fermi Gamma-ray Space Telescope, launched in 2008 June, is a pair-tracking $\gamma$-ray telescope, detecting photons in the range of $\sim 20 \mathrm{MeV}$ to more than $300 \mathrm{GeV}$. Details of the LAT instrument and the pre-launch performance expectations can be found in Atwood et al. (2009), and the on-orbit calibration is described in Abdo et al. (2009a). Thanks to its wide field of view, the Fermi-LAT is an ideal telescope to study Galactic diffuse $\gamma$ rays. Past studies of Galactic diffuse emission by Fermi-LAT can be found in, e.g., Ackermann et al. (2012a), and Casandjian (2015).

Routine science operations with the LAT started on 2008 August 4. We have accumulated events from 2008 August 4 to 2015 August 2 (i.e., 7 years) to study diffuse $\gamma$ rays in our ROI. During most of this time interval, the LAT was operated in sky survey mode, obtaining complete sky coverage every two orbits and relatively uniform exposure over time. We used the Pass 8 event data, and used the standard LAT analysis software, Fermi Science Tools ${ }^{4}$ version v10r00p05, to select events satisfying the so-called Clean class in order to obtain low-background events. We also required that the reconstructed zenith angles of the arrival direction of the photons be less than $100^{\circ}$ to reduce contamination by photons from the Earth atmosphere. In addition, we excluded the periods of time during which the LAT detected bright $\gamma$-ray bursts or solar flares. (The integrated period of time excluded in this procedure is negligible compared to that excluded to remove data with flares of $3 \mathrm{C} 454.3$, described below) We also referred to the Monitored Source List light curves ${ }^{5}$, and excluded the periods of time (300 days in total) during which the LAT detected flares of 3C 454.3. This reduced the contamination from the bright active galactic nucleus in diffuse emission modeling while keeping $\sim 90 \%$ of the good time interval. We used the latest response functions that match our dataset and event selection, P8R2_CLEAN_V6, in the following analysis. Although we did not take into account the energy dispersion ${ }^{6}$ in the analysis, the impact on the results is expected to be small since we analyzed the data above $0.3 \mathrm{GeV}$ as described in Section 4.1.

\subsection{Model to Represent the Gamma-ray Emission}

We modeled the $\gamma$-ray emission observed by Fermi-LAT as a linear combination of the gas column density model map(s) constructed from the Planck dust map, IC emission, isotropic component and $\gamma$-ray point sources. The use of the gas column density maps as a template is based on the assumption that $\gamma$ rays are generated via interactions between the CRs and ISM gas and that CR densities do not vary significantly over the scale of the interstellar complexes in this study. This assumption is simple but very plausible, particularly in high-Galactic latitude regions, such as the one studied here. We started with a single $N\left(\mathrm{H}_{\text {tot }}\right)$ map based on Planck dust model maps $\left(R\right.$ or $\left.\tau_{353}\right)$ in Section 4.1 and employed multiple $N\left(\mathrm{H}_{\text {tot }}\right)$ maps sorted by $T_{\mathrm{d}}$ in Section 4.2 . We note that our $N\left(\mathrm{H}_{\text {tot }}\right)$ model map traces not only atomic but also molecular hydrogen since dust is expected to be well mixed with the ISM gas in both phases. We also included an IC model map and models for point sources. To model the $\gamma$ rays produced via IC scattering, we used GALPROP ${ }^{7}$ (e.g., Strong \& Moskalenko 1998; Strong et al. 2007), a numerical code that solves the CR transport equation within the Galaxy and predicts the $\gamma$-ray emission produced via the interactions of CRs with interstellar matter and low-energy photons (IC scattering). The IC emission is calculated from the distribution of propagated electrons and the interstellar radiation field developed by Porter et al. (2008). Here, we adopted the IC model map produced in the GALPROP run 54_77Xvarh7S, which was used in LAT collaboration publications ${ }^{8}$ such as Ackermann et al. (2011), as our baseline model. Since the integrated intensity (over the solid angle) of the IC emission is lower than that of the isotropic component (see below) and the gas-related diffuse $\gamma$ rays in the region studied (see Figure 7), the specific choice of the IC model does not affect the obtained results significantly. The effect of the IC model uncertainty is examined in Section 4.2. To model the individual $\gamma$-ray sources, we referred to the third Fermi-LAT catalog (3FGL) described in Acero et al. (2015), which is based on the first four years of the science phase of the mission and includes more than 3000 sources detected at a significance of $\geq 4 \sigma$. For our analysis we considered 57 3FGL sources (detected at a significance of $\geq 5 \sigma^{9}$ ) in our ROI, and 17 bright sources $(\geq 20 \sigma)$ just outside it (within $\left.10^{\circ}\right)$ to take account of their possible contamination. We also included 3FGL J2338.7+0251 (4.8 $\sigma$

\footnotetext{
4 http://fermi.gsfc.nasa.gov/ssc/data/analysis/software/

5 http://fermi.gsfc.nasa.gov/ssc/data/access/lat/msl_lc/

6 http://fermi.gsfc.nasa.gov/ssc/data/analysis/documentation/Pass8_edisp_usage.html

7 http://galprop.stanford.edu

8 https://www-glast.stanford.edu/cgi-bin/pubpub
}

9 As described in Section 4.1, we iteratively included sources in several groups at a time in the order of decreasing significance, and confirmed that including sources with significance from $5 \sigma$ to $6 \sigma$ did not affect the gas-related scale factors significantly. We therefore did not include sources with lower 3FGL significance. 
detection in 3FGL) and a source located at $(l, b)=(71.75,-53.5)$ which became brighter after the period of time investigated in 3FGL. The position of the latter source was determined by visual inspection of the $\gamma$-ray map and was fixed in the analysis. We also added an isotropic component to represent the extragalactic diffuse emission and the residual instrumental background from misclassified CR interactions in the LAT detector. Another possible source of diffuse $\gamma$-ray emission is $\mathrm{CR}$ interactions with ionized gas. In order to estimate its contribution, we referred to Casandjian (2015) and used the free-free intensity map at a frequency of $22.7 \mathrm{GHz}$ extracted from 9 year of $W M A P$ observations (Bennett et al. 2013) as a template for the $\gamma$-ray emission correlated with ionized hydrogen. We used the scaling factor adopted by Casandjian (2015) and found that the estimated column density is at most $\sim 10^{20} \mathrm{~cm}^{-2}$ at three spots in our ROI. Two of them are positionally coincident with the two brightest $\gamma$-ray sources in our ROI, 3C 454.3 and 3FGL 2232.5+1143. The third spot is positionally coincident with localized residuals seen in our $\gamma$-ray count map [Figure 8a; $(l, b) \sim(63.7,-34.2)$ ]. Therefore we can securely expect that the impact of the ionized gas on the determination of the neutral gas component is minimal and we did not take the ionized gas into account in our analysis.

Then, $\gamma$-ray intensities $I_{\gamma}(l, b, E)\left(\mathrm{ph} \mathrm{s}^{-1} \mathrm{~cm}^{-2} \mathrm{sr}^{-1} \mathrm{MeV}^{-1}\right)$ can be modeled as

$$
I_{\gamma}(l, b, E)=\sum_{i} c_{1, i}(E) \cdot q_{\gamma}(E) \cdot N\left(\mathrm{H}_{\mathrm{tot}}\right)_{i}(l, b)+c_{2}(E) \cdot I_{\mathrm{IC}}(l, b, E)+I_{\mathrm{iso}}(E)+\sum_{j} \operatorname{PS}_{j}(l, b, E)
$$

where $N\left(\mathrm{H}_{\text {tot }}\right)_{i}$ is the total gas column density model $\left(\mathrm{cm}^{-2}\right)$ map $(\mathrm{s})$ in either atomic or molecular phase, $q_{\gamma}(E)$ $\left(\mathrm{ph} \mathrm{s}^{-1} \mathrm{sr}^{-1} \mathrm{MeV}^{-1}\right.$ ) is the differential $\gamma$-ray yield or $\gamma$-ray emissivity per $\mathrm{H}$ atom, $I_{\mathrm{IC}}(l, b, E)$ and $I_{\text {iso }}(E)$ are the IC model and isotropic background intensities $\left(\mathrm{ph} \mathrm{s}^{-1} \mathrm{~cm}^{-2} \mathrm{sr}^{-1} \mathrm{MeV}^{-1}\right)$, respectively, and $\operatorname{PS}_{j}(l, b, E)$ represents the point source contributions. The subscript $i$ allows for the separation of $N\left(\mathrm{H}_{\text {tot }}\right)$ maps by $T_{\mathrm{d}}$ (Section 4.2$)$. We applied the $\gamma$-ray emissivity model for the local interstellar spectrum (LIS) of CRs and the so-called nuclear enhancement factor $\epsilon_{\mathrm{M}}$ (a scale factor to take account of the effect of heavy nuclei in both CRs and the target matter) of 1.84 (Mori 2009) adopted by Abdo et al. (2009b). To accommodate the uncertainties in the LIS and $\epsilon_{\mathrm{M}}$, we included scale factors $\left[c_{1, i}(E)\right.$ in Equation (1)] as free parameters. It will be 1 if the measured $\gamma$-ray emissivity agrees with the LIS and $\epsilon_{\mathrm{M}}$ we adopted. The IC emission model (see above) also is uncertainly known, and we included another scale factor $\left[c_{2}(E)\right.$ in Equation (1)] as a free parameter. The isotropic component $I_{\text {iso }}$ and the point source contributions were also taken to be free parameters as a function of energy. The positions of sources were fixed to the values in 3FGL. We divided $\gamma$-ray data into several energy ranges and fit Equation (1) to $\gamma$-rays in each energy range using the binned likelihood method implemented in Fermi Science Tools. When using multiple $N\left(\mathrm{H}_{\text {tot }}\right)$ maps (Section 4.2 and Appendix C), we used wider energy ranges and modeled $c_{2}(E)$ with a power law function as $c_{2}(E)=c_{2 \mathrm{n}} \cdot\left(E / E_{0}\right)^{c_{2 \mathrm{i}}}$ where $E_{0}$ is a reference energy. 


\section{DATA ANALYSIS}

\section{1. $\quad$ Initial Modeling with a Single Gas Map}

We started our data analysis using a single total gas column density model maps based on $R$ or $\tau_{353}$. To construct the $N\left(\mathrm{H}_{\text {tot }}\right)$ model maps, we assumed a proportionality between $N\left(\mathrm{H}_{\text {tot }}\right)$ and $R$ (or $\tau_{353}$ ) and that $\mathrm{HI}$ is optically thin and well represents the total gas column density at least for regions with the high-temperature areas $\left(T_{\mathrm{d}} \geq 21.5 \mathrm{~K}\right)$. First we made least-squares fit to the $T_{\mathrm{d}} \geq 21.5 \mathrm{~K}{ }^{10}$ dust- $W_{\mathrm{H} \text { I }}$ relation in Figure 2 with a linear function with an intercept fixed at $0^{11}$ and obtained coefficients of $(19.8 \pm 1.5) \times 10^{8} \mathrm{~K} \mathrm{~km} \mathrm{~s}^{-1}\left(\mathrm{~W} \mathrm{~m}^{-2} \mathrm{sr}^{-1}\right)^{-1}$ and $(102 \pm 13) \times 10^{6} \mathrm{~K} \mathrm{~km} \mathrm{~s}^{-1}$ for the $R$ and $\tau_{353}$, respectively, where the errors are given as the RMS deviations. We then converted $R$ (or $\tau_{353}$ ) into $N\left(\mathrm{H}_{\text {tot }}\right)$ maps, using the coefficients obtained and multiplied by $1.82 \times 10^{18} \mathrm{~cm}^{-2}\left(\mathrm{~K} \mathrm{~km} \mathrm{~s}^{-1}\right)^{-1}$. The obtained total gas column density template maps (proportional to the $R$ or $\tau_{353}$ maps) are shown in Figure 3 . By comparing these maps to the $N\left(\mathrm{HI}_{\text {thin }}\right)$ map and the $W_{\mathrm{CO}}$ maps shown in Figure 1, we can recognize dense gas not accounted for by H I (the optically thin case) in the MBM 53, 54, and 55 clouds and the Pegasus loop near the emission from CO. We can also see that the $\tau_{353}$-based map predicts a stronger contrast for $N\left(\mathrm{H}_{\text {tot }}\right)$ distribution, and approximately a factor of two higher gas column density in dense clouds when compared to the $R$-based map.

Because good angular resolution is essential to examine the correlation between the $\gamma$ rays and the gas distribution, we restricted the energy to above $0.3 \mathrm{GeV}$. The model described in Equation 1 was fitted to the data using Fermi Science Tools, which take into account the energy-dependent instrumental point-spread function and the effective area. We analyzed the LAT data from 0.3 to $72.9 \mathrm{GeV}$ using the logarithmically equally spaced energy bands $0.3-0.52 \mathrm{GeV}$, 0.52-0.9 GeV, 0.9-1.56 GeV, 1.56-2.7 GeV, 2.7-4.68 GeV, and 4.68-8.1 GeV. Above 8.1 GeV, we used wider energy ranges of $8.1-24.3 \mathrm{GeV}$ and $24.3-72.9 \mathrm{GeV}$ to compensate for the low photon statistics. We then have compared the data and model in each energy range using a binned maximum-likelihood method with Poisson statistics in $0.25 \times 0.25$ bins. Within each narrow energy range, we assumed constant spectra for the gas component and the IC emission and assumed $c_{1}$ and $c_{2}$ to be free normalization parameters. For $I_{\text {iso }}$ and $\mathrm{PS}_{j}$ we assumed power-law spectra with photon index fixed at 2.2 and free normalization. In the highest energy range $(24.3-72.9 \mathrm{GeV})$, we found that the IC component (less intense than the isotropic component) was not well determined and fixed the scale factor to 1 . When modeling the point sources, we iteratively included them in several groups at a time in the order of decreasing significance. We first included and fitted nine bright sources detected in 3FGL at more than $20 \sigma$; then added and fit a second group (nine sources) detected at 13-20 $\sigma$, freezing the source parameters already included; and added/fit a third group (10

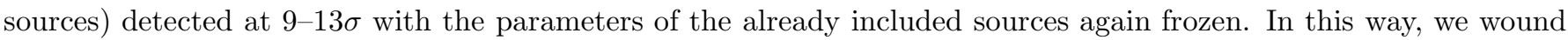
down to the sources detected at more than $5 \sigma$ in 3FGL. In each step, the parameters of the diffuse emission model were always kept free to be varied. We found that by including sources with significance from $5 \sigma$ to $6 \sigma$, the effects on gas-related scale factors were 1-2\% (comparable to or smaller than the statistical error) below $8.1 \mathrm{GeV}$, and $\sim 4 \%$ (about one-fourth of the statistical error) in the highest energy bin. We therefore did not include sources with lower 3FGL significance. Finally, the analysis was repeated with all the sources, letting only the parameters of the diffuse model and those of the nine brightest sources vary freely. To model the contamination from outside the ROI, we took into account 17 point sources (with model parameters fixed to those of 3FGL) detected above $20 \sigma$ in 3FGL located at a distance $\leq 10^{\circ}$ from the region boundaries. We also used $N\left(\mathrm{H}_{\mathrm{tot}}\right)$ and $I_{\mathrm{IC}}$ maps including peripheral regions. The obtained log-likelihoods, $\ln L^{12}$ summed over individual energy ranges in $0.3-72.9 \mathrm{GeV}$ with the $R$-based and $\tau_{353}$-based $N\left(\mathrm{H}_{\text {tot }}\right)$ maps are 757496.7 and 757452.3 , respectively. Therefore, the $R$-based $N\left(\mathrm{H}_{\text {tot }}\right)$ map is preferred by the $\gamma$-ray data. The average of the normalization for the gas component, $c_{1}$ in Equation 1, is $0.884 \pm 0.011$ and $0.391 \pm 0.005$ for the $R$-based and $\tau_{353}$-based maps, respectively.

10 Later we confirmed that the coefficient for $R$ was unchanged when we broadened the temperature range to $T_{\mathrm{d}} \geq 20.5 \mathrm{~K}$ in Section 4.3 . We also evaluated the systematic uncertainty of the coefficient and its effect on the H I emissivity spectrum in Section 5 .

11 Whether we allow the intercept to be free to vary or hold it fixed at 0 (or a small value) is not expected to affect the results significantly, since we determined the coefficient in regions with high $T_{\mathrm{d}}$ where the scatter is narrow, and then applied the correction based on $T_{\mathrm{d}}$ [Equation (3)] to match the $\gamma$-ray data.

$12 L$ is conventionally calculated as $\ln L=\sum_{i} n_{i} \ln \left(\theta_{i}\right)-\sum_{i} \theta_{i}$, where $n_{i}$ and $\theta_{i}$ are the data and the model-predicted counts in each pixel denoted by the subscript, respectively (see, e.g., Mattox et al. 1996) 


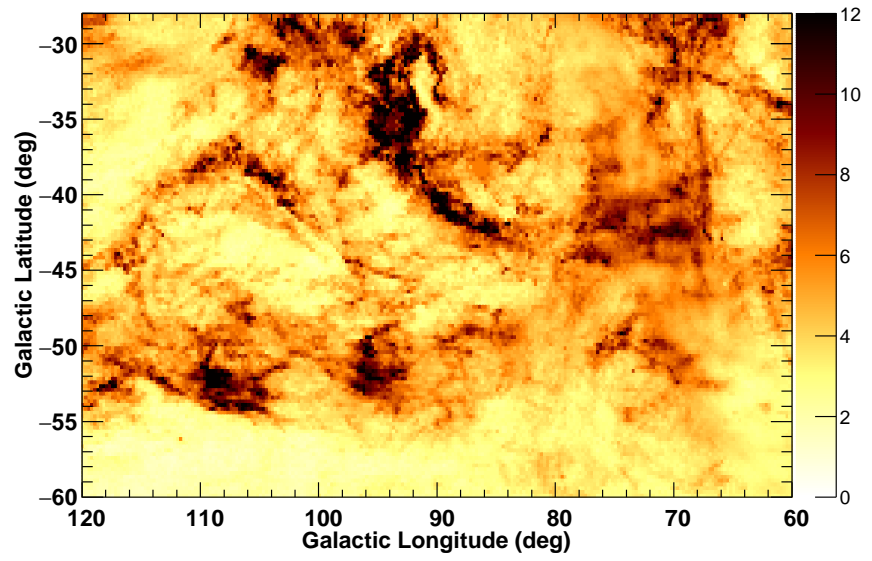

(a)

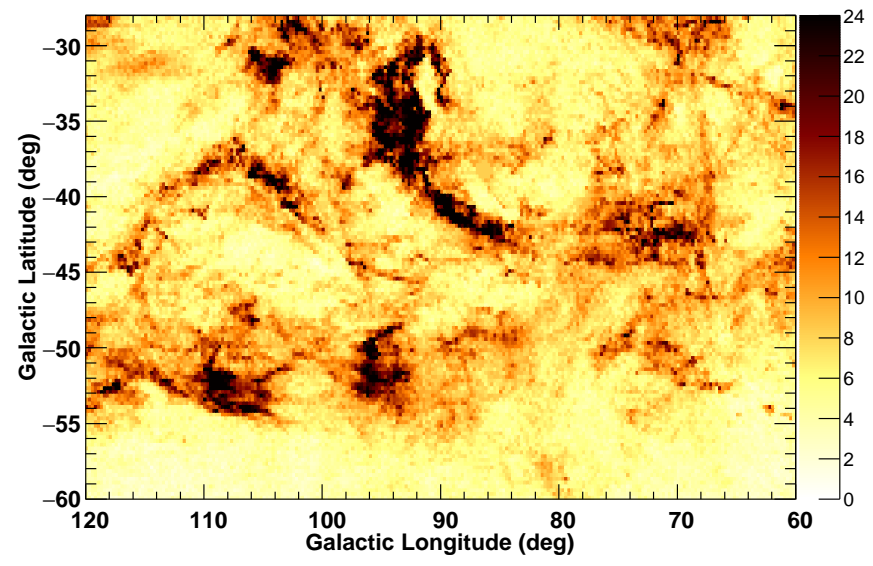

(b)

Figure 3. The total gas column density template maps shown in units of $10^{20} \mathrm{~cm}^{-2}$ : (a) map based on $R\left(N\left(\mathrm{H}_{\text {tot }}\right) \propto R\right)$ and (b) map based on $\tau_{353}\left(N\left(\mathrm{H}_{\text {tot }}\right) \propto \tau_{353}\right)$. See the text in Section 4.1 for details of the procedure to construct those maps. Note that the dynamic range is different in the two plots. 


\subsection{Dust Temperature-Sorted Modeling}

As we saw in Section 2 (Figure 2), the correlations between $W_{\mathrm{H}_{\mathrm{I}}}$ and dust tracers $\left(R\right.$ or $\left.\tau_{353}\right)$ depend on $T_{\mathrm{d}}$, and the temperature dependence is significantly different in the cases of $R$ or $\tau_{353}$. Even though the $R$-based $N\left(\mathrm{H}_{\text {tot }}\right)$ map is preferred to the $\tau_{353}$-based one in terms of $\ln L$ by $\gamma$-ray data analysis as described in Section 4.1 , the true $N\left(\mathrm{H}_{\text {tot }}\right)$ distribution could be appreciably different from either of them. To investigate the temperature dependence more quantitatively, we proceeded to an analysis with $T_{\mathrm{d}}$-sorted template maps as described below.

We split the $N\left(\mathrm{H}_{\text {tot }}\right)$ template map (constructed from $R$ or $\tau_{353}$ ) into four based on $T_{\mathrm{d}}$, for $T_{\mathrm{d}} \leq 18 \mathrm{~K}, T_{\mathrm{d}}=18-19 \mathrm{~K}$, $T_{\mathrm{d}}=19-20 \mathrm{~K}$ and $T_{\mathrm{d}} \geq 20 \mathrm{~K}^{13}$, and fit $\gamma$-ray data with Equation 1 using the four template maps, with scaling factors $\left(c_{1, i}(E)\right.$ for each of the four templates) freely varying individually instead of using a single $N\left(\mathrm{H}_{\text {tot }}\right)$ map. Because our new model now had more free parameters, the narrow energy ranges were no longer feasible and we combined two adjacent energy ranges to make broader ranges: $0.3-0.9 \mathrm{GeV}, 0.9-2.7 \mathrm{GeV}, 2.7-8.1 \mathrm{GeV}$ and $8.1-72.9 \mathrm{GeV}$. To accommodate these wider energy ranges, we modeled the IC (scale factor $c_{2}(E)$ ) and isotropic $\left(I_{\text {iso }}(E)\right)$ intensities as power laws with both the normalization and photon index free to vary in each energy range. Two bright sources, 3C 454.3 and 3FGL J2232.5+1143, were also fitted with power laws with both the normalization and photon index allowed to vary. To test whether splitting the gas template map and fitting the scale factors individually yielded statistically significant improvement in the likelihood, we first fit the $\gamma$-ray data using a single $N\left(\mathrm{H}_{\text {tot }}\right)$ map in the wider energy range, and then proceeded to the analysis with the four template gas maps. The values of $\ln L$ obtained from a fit in four energy bands with a single $N\left(\mathrm{H}_{\text {tot }}\right)$ map (summed over the individual energy ranges) were 1262782.5 and 1262751.2 for the $R$-based and $\tau_{353}$-based maps, respectively, and the values of $\ln L$ obtained from a fit with the four template maps were 1262821.1 and 1262802.8, respectively. The improvement in the fit, the likelihood test statistic TS $\equiv 2 \Delta \ln L$, was 77.2 and 103.2 with 12 more degrees of freedom (giving a statistical significance of $6.7 \sigma$ and $8.2 \sigma$ ) for the $R$-based and $\tau_{353}$-based fits, respectively. Therefore, the fit improvement was significant in both cases $^{14}$; however, the $R$-based analysis was still preferred. Obtained fit parameters and spectra of each component are summarized in Appendix C.

As shown in Tables $\mathrm{C} 2$ and $\mathrm{C} 3$ in Appendix $\mathrm{C}$, we observed that the scaling factors $c_{1, i}(E)$ depended on $T_{\mathrm{d}}$; the averages over the entire energy range are summarized in Figure 4, which shows a clear negative correlation (lower scaling factor at higher $\left.T_{\mathrm{d}}\right)$ and a positive correlation for the $R$-based and $\tau_{353}$-based $N\left(\mathrm{H}_{\text {tot }}\right)$ maps, respectively. These trends cannot be interpreted as being due to the properties of CRs, because the physical environments that determine the $T_{\mathrm{d}}$ (e.g., the ISRF intensity and dust cross section) do not affect the CR density. The only possible explanation in terms of the CR properties is the exclusion of charged particles in dense clouds with large magnetic fields. However, CRs have been confirmed to penetrate into dense cloud cores with $W_{\mathrm{CO}} \geq 10 \mathrm{~K} \mathrm{~km} \mathrm{~s}^{-1}$ (e.g., Abdo et al. 2010; Ackermann et al. 2011, 2012b) which corresponds to densities much larger than those of clouds studied here. Therefore, the main cause of $T_{\mathrm{d}}$ dependence found here is not attributable to the properties of CRs.

Another possible cause of the apparent $T_{\mathrm{d}}$ dependence is the uncertainty of the IC model. Even if we adjusted the IC spectrum by scaling it in each energy range, the spatial distribution of our IC model might not be accurate. This could affect the results shown in Figure 4 in two ways: one by changing the slope of $T_{\mathrm{d}}$ dependence [i.e., changing the measured $T_{\mathrm{d}}$ dependence of $N\left(\mathrm{H}_{\mathrm{tot}}\right) / R$ and $\left.N\left(\mathrm{H}_{\mathrm{tot}}\right) / \tau_{353}\right]$, and the other by changing the values of the scaling factor (i.e., measured $\gamma$-ray emissivity or CR density). To investigate this possibility, we tested several other IC models. As described in Section 2, we used the IC model produced in the GALPROP run 54_77Xvarh7S as our baseline model. This configuration assumes a CR source distribution proportional to

$$
f(r)=\left(\frac{r}{r_{\odot}}\right)^{1.25} \exp \left(-3.56 \cdot \frac{r-r_{\odot}}{r_{\odot}}\right),
$$

where $r$ is the Galactocentric distance and $r_{\odot}=8.5 \mathrm{kpc}$ is the distance from the Sun to the Galactic center. In the baseline model, the boundary of the cosmic-ray halo $z_{h}$ is chosen to be $4 \mathrm{kpc}$ with uniform spatial diffusion coefficient $D_{x x}=\beta D_{0}(\rho / 4 \mathrm{GV})^{\delta}$ across the Galaxy, where $\beta \equiv v / c$ is the velocity of a particle relative to the speed of light, $\rho$ is the rigidity of the particle, and $D_{0}=5.8 \times 10^{28} \mathrm{~cm}^{2} \mathrm{~s}^{-1}$ and $\delta=0.33$ (the Kolmogorov spectrum) were adopted. As described in Ackermann et al. (2011) and de Palma et al. (2012), of those parameters, the CR source distribution and the halo height typically most strongly affect the propagated CR spatial distribution (in Galactocentric distance and the height from the Galactic plane) and therefore the IC spatial distribution (in $l$ and $b$ ). Therefore, we tested

\footnotetext{
13 The relative solid angles in our ROI are $9.5 \%, 41.2 \%, 36.7 \%$, and $12.6 \%$ for $T_{\mathrm{d}} \leq 18 \mathrm{~K}, T_{\mathrm{d}}=18-19 \mathrm{~K}, T_{\mathrm{d}}=19-20 \mathrm{~K}$ and $T_{\mathrm{d}} \geq 20 \mathrm{~K}$, respectively.

14 TS for the null hypothesis is asymptotically distributed as chi-square with the degrees of freedom. (http://fermi.gsfc.nasa.gov/ssc/data/analysis/documentation/Cicerone/Cicerone_Likelihood/Likelihood_overview.html)
} 
two more CR source distributions, the pulsar-based distribution and the SNR (supernova remnant)-based distribution (see figure 12 of Ackermann et al. 2011) in addition to the distribution in the baseline model, and two more CR halo heights, $10 \mathrm{kpc}$ and $20 \mathrm{kpc}$, in addition to the halo height $(4 \mathrm{kpc})$ in the baseline model. To match the direct $\mathrm{CR}$ measurements at Earth, $D_{0}=5.8 \times 10^{28} \mathrm{~cm}^{2} \mathrm{~s}^{-1}$ was adjusted when changing $z_{h}$ as described in Ackermann et al. (2011). The obtained IC maps show the smallest gradient in the Galactic longitude direction with the SNR-based CR source distribution (the flattest distribution of our three choices), and the smallest gradient in the Galactic latitude direction with $z_{h}=20 \mathrm{kpc}$ (the largest halo height of our three choices). Our baseline model provides a reasonably good fit to the data and provides the second highest value of $\ln L$ among all models considered. All nine configurations show the same trend in the $T_{\mathrm{d}}$ dependence of the scale factor $c_{1, i}$ (negative/positive correlation with $T_{\mathrm{d}}$ in the $R$ based $/ \tau_{353}$-based analysis), and the scale factors are not affected significantly. Therefore, we conclude that our finding concerning the $T_{\mathrm{d}}$ dependence is robust against variations in the models of IC emission, and that the primary cause of the dependence is the non-uniformity of $N\left(\mathrm{H}_{\text {tot }}\right) / R$ and $N\left(\mathrm{H}_{\text {tot }}\right) / \tau_{353}$.

One may also argue that $N\left(\mathrm{H}_{\text {tot }}\right) / R$ or $N\left(\mathrm{H}_{\text {tot }}\right) / \tau_{353}$ is appreciably different in dense molecular cloud complexes from that in translucent clouds, affecting the slope of the $T_{\mathrm{d}}$ dependence (and the values of the scale factor if the best-fit isotropic component and IC emission is affected) appreciably. Therefore, we masked areas around the dense molecular clouds traced by CO, as indicated by the dotted lines in Figure 1b, and repeated the same analysis using our baseline IC model. We again confirmed that the $T_{\mathrm{d}}$ dependence we found (negative/positive correlation with $T_{\mathrm{d}}$ in the $R$-based $/ \tau_{353}$-based analyses) remained and the scale factors were not affected significantly. We also masked IVCs in our ROI (see Section 2 and Appendix B) as indicated by the dotted lines in Figure B1b, since they could have different CR density and/or different properties of the ISM gas and dust, and performed the same test. Again, the effect on the results shown in Figure 4 was found to be small. To gauge systematic uncertainties, we bracketed the $T_{\mathrm{d}}$ dependence of our baseline model in Figure 4 with that obtained using the IC model of the pulsar-based CR source distribution and $z_{h}=20 \mathrm{kpc}$ (which shows the largest difference among the nine possibilities from our baseline IC model), that obtained using our baseline IC model but with areas around the molecular clouds traced by CO masked, and that obtained using our baseline IC model but with areas of IVCs masked, as shown by the shaded bands in Figure 4 .

We thus found that the ratios of $N\left(\mathrm{H}_{\mathrm{tot}}\right) / R$ and $N\left(\mathrm{H}_{\mathrm{tot}}\right) / \tau_{353}$ (both $\propto c_{1, i}$ ) are not uniform and depend on $T_{\mathrm{d}}$. Even though the variation is by only $30 \%-40 \%$ over the range of $T_{\mathrm{d}}$ in the region, this shows both $R$ and $\tau_{353}$ are not accurate tracers of the total gas column densities and we give possible explanations for this below.

\section{$R$ (Radiance):}

Under the assumption of a uniform dust-to-gas ratio and dust emissivity, $R$ (the dust bolometric luminosity) per $\mathrm{H}$ atom, or the dust specific luminosity, will be constant if the ISRF is uniform along the line of sight. This is the basis of the claim by the Planck Collaboration (2014) that $R$ is a good tracer of the total dust (and gas) column density. However, even though the ISRF is uniform in the vicinity of the solar system, the $R$ per $\mathrm{H}$ atom could decrease as the gas (and dust) density increases, because the ISRF is more strongly absorbed by dust at higher density. This will cause a correlated decrease in the $T_{\mathrm{d}}$ and dust specific luminosity. This qualitative argument is supported by theoretical works, e.g., by Ysard et al. (2015). We note that results by Ysard et al. (2015) are for very diffuse ISM $\left(N\left(\mathrm{H}_{\text {tot }}\right) \leq 2.5 \times 10^{20} \mathrm{~cm}^{-2}\right)$. Therefore direct comparison with our results is not appropriate. We also note that a correlated decrease in the $T_{\mathrm{d}}$ and dust specific luminosity was observed by Planck Collaboration (2015) in the Chamaeleon molecular cloud region (see Figure 13 of the reference). Therefore, the trend we found is not specific to the region under study here but likely to be universal.

$\tau_{353}:$

In the optically thin limit, the specific intensity of the dust emission $I_{\nu}$ is given as $I_{\nu}=\tau_{\nu} B_{\nu}\left(T_{\mathrm{d}}\right)=$ $\sigma_{\nu} N\left(\mathrm{H}_{\text {tot }}\right) B_{\nu}\left(T_{\mathrm{d}}\right)$, where $\tau_{\nu}$ and $\sigma_{\nu}$ are the optical depth and the dust opacity (cross section) per hydrogen atom, respectively, and $B_{\nu}$ is the Planck function. $\sigma_{\nu}$ depends on the frequency and is often described as a power law, giving $I_{\nu}=\tau_{\nu_{0}}\left(\nu / \nu_{0}\right)^{\beta} B_{\nu}\left(T_{\mathrm{d}}\right)$ (i.e., a modified blackbody) with $\beta=1.5-2$ and $\nu_{0}$ is a reference frequency. Therefore, if the dust cross section is uniform in the ROI, $\tau_{\nu}$ is proportional to $N\left(\mathrm{H}_{\text {tot }}\right)$ and we can measure the total gas column density by measuring the dust optical depth at any frequency. Even though this assumption has been adopted in multiple studies, the dust spectral index $\beta$ and therefore the dust opacity are not uniform but rather anti-correlate with $T_{\mathrm{d}}$ as reported by the Planck Collaboration (2014). We examined our map and confirmed this anti-correlation between $T_{\mathrm{d}}$ and $\beta$ in our ROI.

Therefore, neither $R$ nor $\tau_{353}$ is guaranteed to be a good tracer of $N\left(\mathrm{H}_{\text {tot }}\right)$. As described above, we used another gas tracer, the $\gamma$-ray data from the Fermi-LAT, and found that both $N\left(\mathrm{H}_{\text {tot }}\right) / R$ and $N\left(\mathrm{H}_{\text {tot }}\right) / \tau_{353}$ (both $\left.\propto c_{1, i}\right)$ were 
not uniform in our ROI, whatever the physical reason may be. Therefore, we propose to use the $\gamma$-ray data as a robust tracer of $N\left(\mathrm{H}_{\mathrm{tot}}\right)$ to compensate for the $T_{\mathrm{d}}$ dependence, as described in Section 4.3 in detail. We note that $\gamma$-ray observations suffer from low photon statistics, and contamination by non-gas-related components, such as IC, isotropic component, and point sources, and therefore cannot determine the gas column density alone. Combining the $\gamma$-ray data and other gas tracers (e.g., dust, H I 21-cm line, and CO 2.6-mm line) is essential to quantify the true gas distribution.

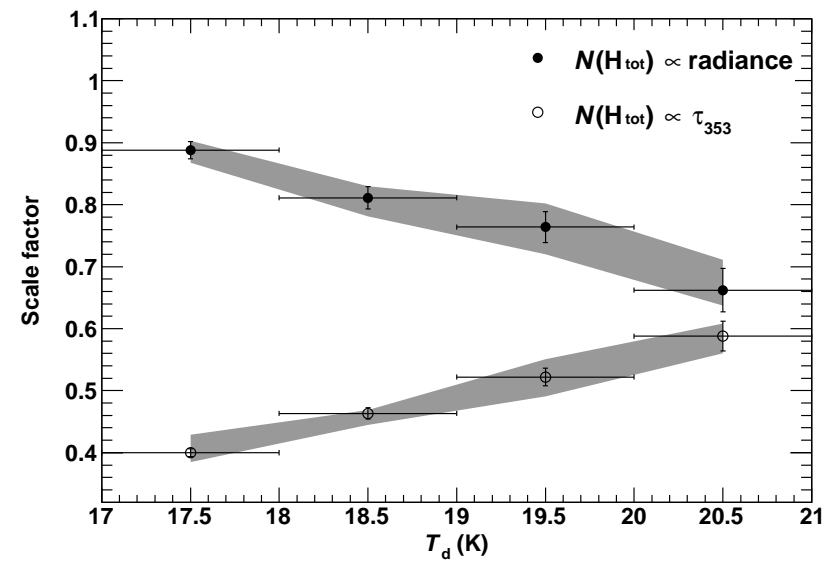

Figure 4. Summary of the scale factors $c_{1, i}$ in Equation (1) averaged over the entire energy range in each range of $T_{\mathrm{d}}$. The filled and open circles show the temperature dependence of the scale factors for the $R$-based and $\tau_{353}$-based $N\left(\mathrm{H}_{\text {tot }}\right)$ maps, respectively, and gray bands show the systematic uncertainty (see the text in Section 4.2 for details). Although a small fraction of the pixels show $T_{\mathrm{d}}$ below $17 \mathrm{~K}$ or above $21 \mathrm{~K}$, they are included in the first or last data points, respectively. 


\subsection{Dust Temperature-Corrected Modeling}

As described in Section 4.2, we found the $T_{\mathrm{d}}$ dependence of the scaling factors of the gas component and concluded that it was primarily due to the dust properties. We aim to use the $\gamma$-ray data as a robust tracer of $N\left(\mathrm{H}_{\text {tot }}\right)$ and apply a correction on the $N\left(\mathrm{H}_{\text {tot }}\right)$ model map to compensate for $T_{\mathrm{d}}$ dependence.

We started from the $R$-based single $N\left(\mathrm{H}_{\text {tot }}\right)$ map (see Figure 3 ) and modified the gas column density with an empirical function as below:

$$
N\left(\mathrm{H}_{\mathrm{tot}, \bmod }\right)=\left\{\begin{array}{l}
N\left(\mathrm{H}_{\mathrm{tot}, \mathrm{R}}\right)\left(T_{\mathrm{d}}>T_{\mathrm{bk}}\right), \\
\left(1+0.05 \cdot C \cdot \frac{T_{\mathrm{bk}}-T_{\mathrm{d}}}{1 \mathrm{~K}}\right) \cdot N\left(\mathrm{H}_{\mathrm{tot}, \mathrm{R}}\right)\left(T_{\mathrm{d}} \leq T_{\mathrm{bk}}\right),
\end{array}\right.
$$

where $T_{\mathrm{bk}}=20.5 \mathrm{~K}$. Above $T_{\mathrm{bk}}$ we retained the original $N\left(\mathrm{H}_{\mathrm{tot}}\right)$ distribution. We re-examined the correlation between $R$ and $W_{\mathrm{H} \text { I }}$ (Figure $2 \mathrm{a}$ ) and confirmed that the coefficient between the two quantities, originally determined for $T_{\mathrm{d}}$ above $21.5 \mathrm{~K}$, was unchanged above $20.5 \mathrm{~K}$. Therefore, our procedure is self-consistent. We tested several choices of coefficients and summarized the value of $\ln L$ in Figure 5 . We found that a coefficient $C$ of 2 (which corresponds to a $10 \%$ apparent decrease in $R$ per $\mathrm{H}$ atom, or $10 \%$ required increase in the gas column density, as $T_{\mathrm{d}}$ decreases by $1 \mathrm{~K}$ ) gave the best representation of the Fermi-LAT data. We also tested $T_{\mathrm{bk}}$ values of $20 \mathrm{~K}$ and $21 \mathrm{~K}$ instead of $20.5 \mathrm{~K}$ in Equation 3, repeated the same analysis, and confirmed that the combination of $T_{\mathrm{bk}}=20.5 \mathrm{~K}$ and $C=2$ gave the largest value of $\ln L$.

Having obtained our modified column density map $N\left(\mathrm{H}_{\mathrm{tot}, \mathrm{mod}}\right)$, we returned to finer energy ranges to study the spectral shape of each component in more detail. The $N\left(\mathrm{H}_{\mathrm{tot}, \mathrm{mod}}\right)$ map and the map of the excess gas column density above $N\left(\mathrm{HI}_{\text {thin }}\right)$ are shown in Figure 6. The fitting parameters and the obtained spectral components are summarized in Table 1 and Figure 7, respectively. The average of the scale factor for the gas component, $c_{1}$, is $0.675 \pm 0.008$ in 0.3-72.9 GeV. The data count map, model count map and data/model ratio map are summarized in Figure 8. By comparing Figure 6b with Figure 1, we can see that the excess gas column density above $N\left(\mathrm{HI}_{\text {thin }}\right)$ is greatest in the MBM 53, 54, and 55 clouds (located at $l=84^{\circ}$ to $96^{\circ}$ and $b=-44^{\circ}$ to $-30^{\circ}$ ) traced by CO emission. We also see a significant amount of excess gas in the Pegasus loop located at $l$ from $98^{\circ}$ to $118^{\circ}$ and $b$ from $-55^{\circ}$ to $-35^{\circ}$, and an area of $l$ from $68^{\circ}$ to $78^{\circ}$ and $b$ from $-44^{\circ}$ to $-40^{\circ}$. They are regions with low $T_{\mathrm{d}}$ as indicated in Figure 1.

Table 1. Results of the fit with a single, corrected $N\left(\mathrm{H}_{\text {tot }}\right)$ map

\begin{tabular}{cccc}
\hline \hline $\begin{array}{c}\text { Energy } \\
(\mathrm{GeV})\end{array}$ & $c_{1}$ & $c_{2}$ & Integ. $I_{\text {iso }}{ }^{\mathrm{a}}$ \\
\hline $0.3-0.52$ & $0.62 \pm 0.01$ & $0.80 \pm 0.10$ & $2.68 \pm 0.05$ \\
$0.52-0.9$ & $0.68 \pm 0.02$ & $0.93 \pm 0.12$ & $1.27 \pm 0.03$ \\
$0.9-1.56$ & $0.71 \pm 0.02$ & $0.92 \pm 0.15$ & $0.60 \pm 0.02$ \\
$1.56-2.7$ & $0.70 \pm 0.02$ & $0.62 \pm 0.19$ & $0.29 \pm 0.01$ \\
$2.7-4.68$ & $0.79 \pm 0.04$ & $0.74 \pm 0.25$ & $0.116 \pm 0.008$ \\
$4.68-8.1$ & $0.76 \pm 0.06$ & $0.93 \pm 0.35$ & $0.056 \pm 0.006$ \\
$8.1-24.3$ & $0.80 \pm 0.09$ & $0.45 \pm 0.41$ & $0.047 \pm 0.005$ \\
$24.3-72.9$ & $0.95 \pm 0.27$ & 1.0 (fixed) & $0.008 \pm 0.001$ \\
\hline
\end{tabular}

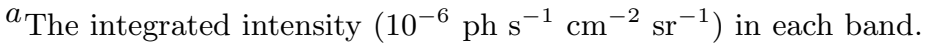

Note - The errors are 1-sigma statistical uncertainties. In each energy bin, $c_{1}$ gives the scale factor of the gas-related component, IC is multiplied by a scaling factor $c_{2}$, and $I_{\text {iso }}$ is modeled with a power law (photon index is fixed to 2.2) with the integrated intensity as a free parameter. 


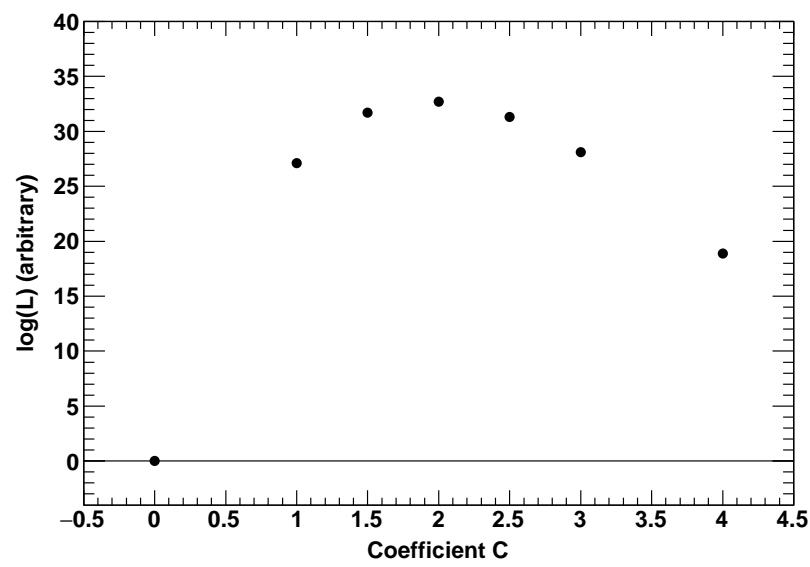

Figure 5. Summary of $\ln L$ as a function of the coefficient $C$ in Equation 3 with $T_{\mathrm{bk}}=20.5 \mathrm{~K}$.

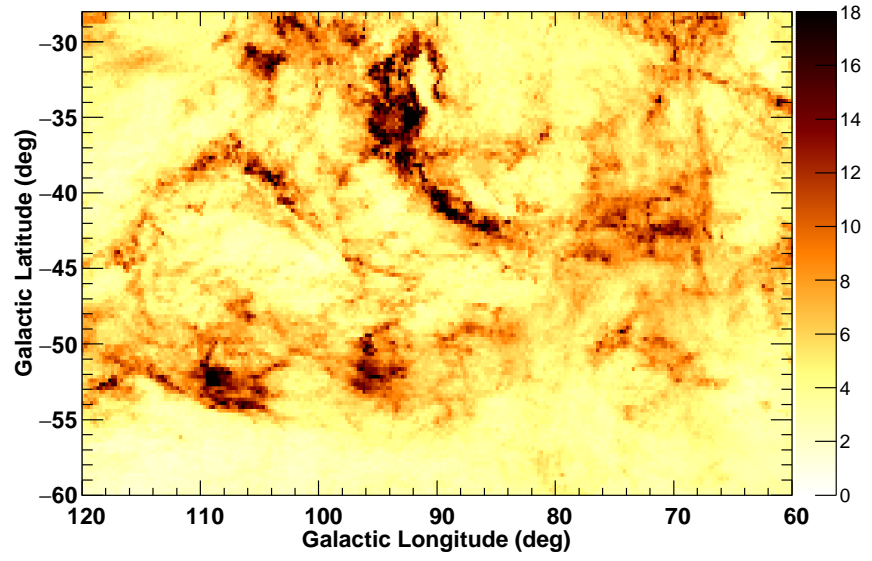

(a)

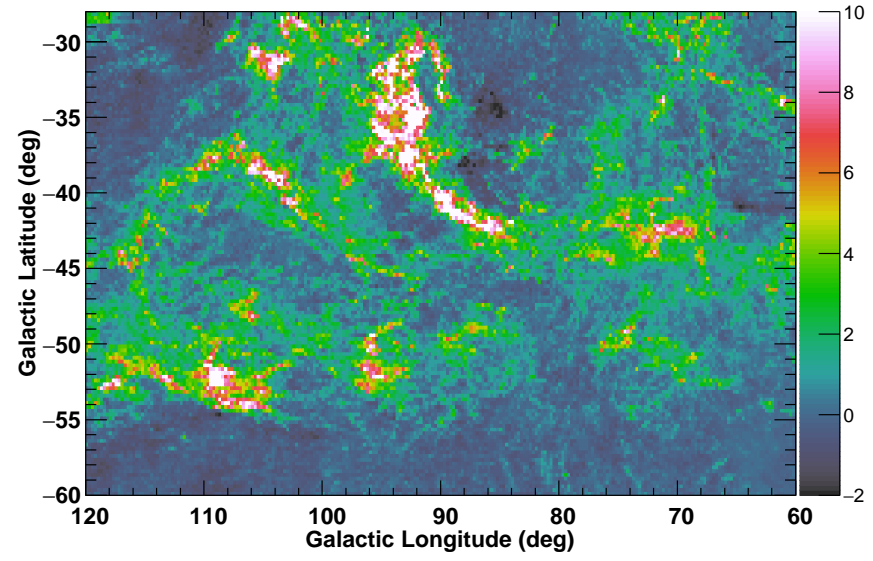

(b)

Figure 6. (a) The corrected $N\left(\mathrm{H}_{\text {tot }}\right)$ map and (b) the map of the excess gas column density above $N\left(\mathrm{HI}_{\mathrm{thin}}\right)$. Both panels are shown in units of $10^{20} \mathrm{~cm}^{-2}$. 


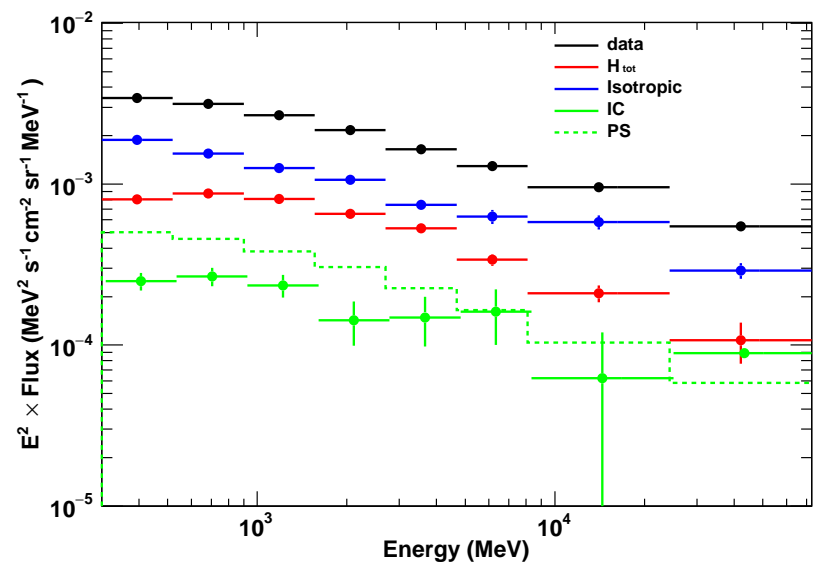

Figure 7. Spectrum of each component obtained by the fit with the corrected $N\left(\mathrm{H}_{\text {tot }}\right)$ map. 


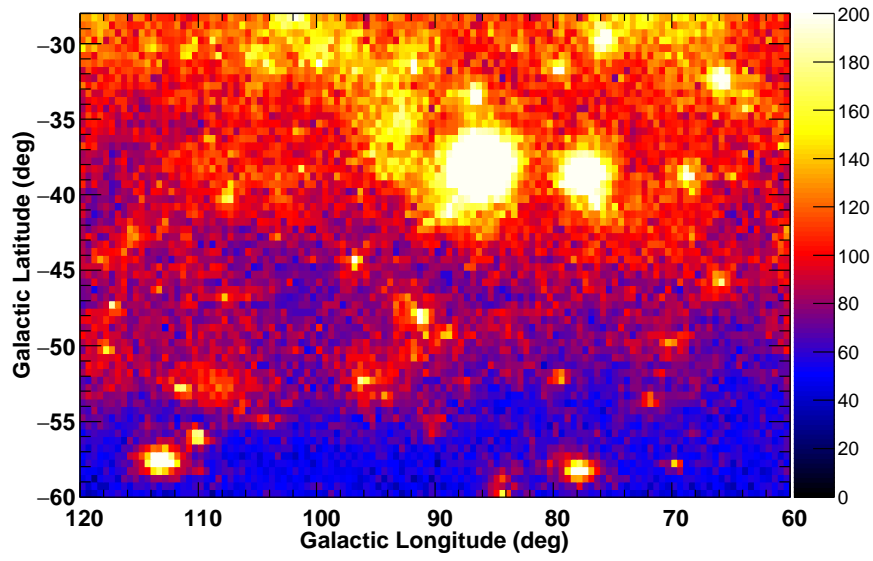

(a)

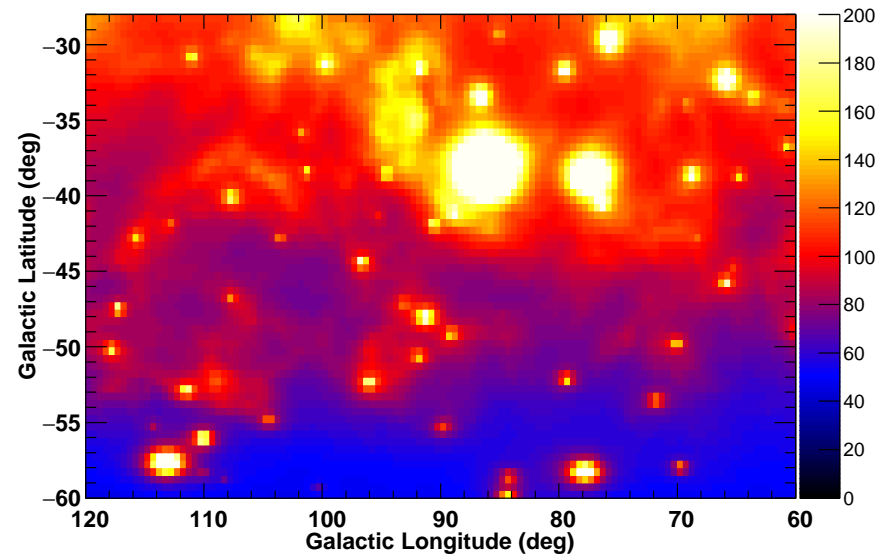

(b)

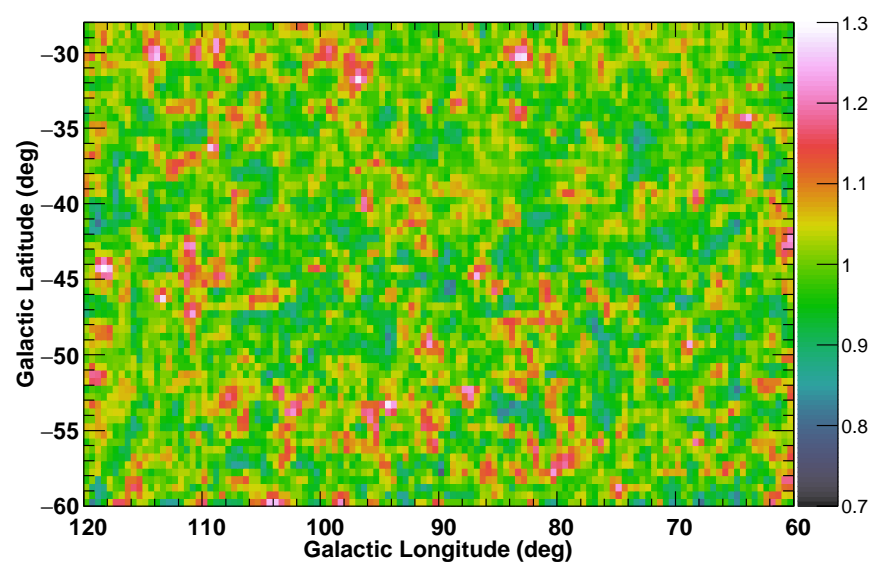

(c)

Figure 8. (a) The data count map, (b) the model count map, and (c) the data/model ratio map obtained by the fit with the corrected $N\left(\mathrm{H}_{\text {tot }}\right)$ map. Although the fit has been performed in $0.25 \times 0.25$ bins, these maps have been rebinned in $0.5 \times 0.5$ pixels for display. Data/model ratio map has been smoothed with a k3a kernel (1-2-1 two-dimensional boxcar smoothing) in ROOT framework (https://root.cern.ch). 


\section{DISCUSSION}

In Section 4 we showed that neither $R$ nor $\tau_{353}$ were good representations of the total gas column density in the ROI and used the Fermi-LAT $\gamma$-ray data to compensate for the $T_{\mathrm{d}}$ dependence of $N\left(\mathrm{H}_{\text {tot }}\right) / R$. The correlation between $W_{\mathrm{H} \text { I }}$ and the $N\left(\mathrm{H}_{\mathrm{tot}}\right)$ inferred by the $\gamma$-ray data analysis obtained from Equation 3 with $T_{\mathrm{bk}}=20.5 \mathrm{~K}$ and $C=2$ is shown in Figure 9a, in which we observe moderate scatter. We note that our corrected $N\left(\mathrm{H}_{\text {tot }}\right)$ model map is based on $R$, and therefore is expected to include contributions from both atomic and molecular hydrogen.

Because the MBM 53, 54, and 55 clouds and the Pegasus loop are located at similar distances from the solar system and most of the $\mathrm{HI}$ clouds are expected to coexist with the $\mathrm{H}_{2}$ clouds (because they are located at high Galactic latitudes), we can estimate the mass of gas from the column density $N(\mathrm{H})$ as

$$
M=\mu m_{\mathrm{H}} d^{2} \int N(\mathrm{H}) d \Omega,
$$

where $d$ is the distance to the cloud, $m_{\mathrm{H}}$ is the mass of the hydrogen atom and $\mu=1.41$ is the mean atomic mass per $\mathrm{H}$ atom (Däppen 2000). Although our ROI includes IVCs, their contribution to the integral of $N(\mathrm{HI})$ was at the 5\% level (see Appendix B); therefore the impact on the discussion of the cloud mass distribution is small. From Equation (4), $\int N(\mathrm{H}) d \Omega=10^{22} \mathrm{~cm}^{-2} \operatorname{deg}^{2}$ corresponds to $\sim 740 \mathrm{M}_{\odot}$ for $d=150 \mathrm{pc}$. The integrated H I column density for the optically thin case, $\int N\left(\mathrm{HI}_{\mathrm{thin}}\right) d \Omega=1.82 \times 10^{18} \cdot \int W_{\mathrm{H} \text { I }} d \Omega$, and the integrated column density of the excess gas $\left(\int\left(N\left(\mathrm{H}_{\text {tot }}\right)-N\left(\mathrm{H}_{\mathrm{thin}}\right)\right) d \Omega\right)$ are calculated in $0.5-\mathrm{K}$ step $T_{\mathrm{d}}$ bins and are summarized in Figure $9 \mathrm{~b}$. We observed that the excess gas starts to appear below $T_{\mathrm{d}}=20 \mathrm{~K}$ and contributes $\sim 1 / 3$ of the total amount of gas in the range of $T_{\mathrm{d}}=18-18.5 \mathrm{~K}$. Below $T_{\mathrm{d}}=18 \mathrm{~K}$, the excess gas is as massive as that of $\mathrm{H}$ I for the optically thin case.

Some fraction of this excess gas is molecular hydrogen traced by $\mathrm{CO}$ (hereafter denoted as $\mathrm{H}_{2, \mathrm{CO}}$ ), and we can calculate the mass as

$$
M=\mu m_{\mathrm{H}} d^{2} \int 2 N\left(\mathrm{H}_{2, \mathrm{CO}}\right) d \Omega=\mu m_{\mathrm{H}} d^{2} \cdot 2 X_{\mathrm{CO}} \int W_{\mathrm{CO}} d \Omega .
$$

To estimate the value of $X_{\mathrm{CO}}$, we examined the correlation between $N\left(\mathrm{H}_{\text {tot }}\right)-N\left(\mathrm{H}_{\mathrm{thin}}\right)$ and (moment-masked) $W_{\mathrm{CO}}$ as shown in Figure 9c. There, we observe large scatter, particularly in the low $W_{\mathrm{CO}}$ area, likely due to dark gas (gas not traced by the H I 21-cm line or the CO $2.6-\mathrm{mm}$ line). If we restrict $T_{\mathrm{d}}$ below $17 \mathrm{~K}$, where the $\mathrm{H}_{2}$ gas traced by CO is expected to be dominant, we see that the correlation becomes better and $W_{\mathrm{CO}}$ starts to appear above $\sim 5 \times 10^{20} \mathrm{~cm}^{-2}$. The average of $N\left(\mathrm{H}_{\text {tot }}\right)-N\left(\mathrm{HI}_{\text {thin }}\right)$ in $T_{\mathrm{d}} \leq 17 \mathrm{~K}$ and $W_{\mathrm{CO}} \leq 0.1 \mathrm{~K} \mathrm{~km} \mathrm{~s}^{-1}$ is $5.4 \times 10^{20} \mathrm{~cm}^{-2}$, which can be interpreted as an offset due to the $\mathrm{H}_{2}$ gas not being traced by $\mathrm{CO}$ or $\mathrm{HI}$ gas in the optically thin condition. We then fit the other data points in $T_{\mathrm{d}} \leq 17 \mathrm{~K}$ using a linear function with its intercept in the horizontal axis fixed at this average, and obtained a slope of $0.40 \mathrm{~K} \mathrm{~km} \mathrm{~s}^{-1}\left(10^{20} \mathrm{~cm}^{-2}\right)^{-1}$. This translates into $X_{\mathrm{CO}}=1.25 \times 10^{20} \mathrm{~cm}^{-2}\left(\mathrm{~K} \mathrm{~km} \mathrm{~s}^{-1}\right)^{-1}$ which is a typical value obtained via the $\gamma$-ray data analysis of nearby molecular cloud complexes by Fermi-LAT (see Figure 9 in Grenier et al. 2015). We note that, given the larger scatter in the $W_{\mathrm{CO}}$ versus $N\left(\mathrm{H}_{\text {tot }}\right)-N\left(\mathrm{H}_{\mathrm{t} \text { thin }}\right)$ relation (at least partially due to the dark gas as described above), the uncertainty of our estimated $X_{\mathrm{CO}}$ is large and possibly by a factor as large as two. Although a precise determination of $X_{\mathrm{CO}}$ is important, it is beyond the scope of this study. Using the $X_{\mathrm{CO}}$ estimated above, we calculated $2 X_{\mathrm{CO}} \int W_{\mathrm{CO}} d \Omega$ which is a measure of the CO-bright $\mathrm{H}_{2}$ mass (Equation 5), and plotted the distribution in Figure 9b. The integral of $N\left(\mathrm{H}_{\text {thin }}\right)$ and $N\left(\mathrm{H}_{\text {tot }}\right)-N\left(\mathrm{HI}_{\text {thin }}\right)$ is 60.9 and 16.5 in units of $10^{22} \mathrm{~cm}^{-2} \mathrm{deg}^{2}$, respectively, and that of $W_{\mathrm{CO}}$ multiplied by $2 X_{\mathrm{CO}}$ is 2.6 in the same units. By comparing the excess mass distribution (red line) and the CO-bright $\mathrm{H}_{2}$ mass distribution (blue dotted line) in Figure 9b, we can see that most of the excess mass can be attributed to CO-bright $\mathrm{H}_{2}$ gas at $T_{\mathrm{d}} \leq 17 \mathrm{~K}$, as expected. However, above $17.5 \mathrm{~K}$, the contribution of the CO-bright molecular mass is $\leq 10 \%$ and cannot explain the excess mass even if we assume $X_{\mathrm{CO}}$ is uncertain by a factor of 2 . This is the so-called "dark gas" and it contributes $(16.5-2.6) / 60.9 \sim 25 \%$ of the mass in $\mathrm{HI}$ in the optically thin case.

The ratio of the mass of dark gas to that of the $\mathrm{H}_{2}$ traced by $\mathrm{CO}$ is $(16.5-2.6) / 2.6 \sim 5$. (The value should be considered as an upper limit since we assumed that HI is optically thin.) This is significantly higher than the values reported by the Planck Collaboration (2011) ( 120\% on average at high Galactic latitude) and that by the Planck Collaboration (2015) ( $\sim 200 \%$ in the Chamaeleon clouds). In other words, the region studied here is very dark-gas-rich when compared to $\mathrm{H}_{2}$ traced by $\mathrm{CO}$. To explain the dark gas as being primarily due to CO-dark $\mathrm{H}_{2}$, the ratio of the CO-dark $\mathrm{H}_{2}$ and the CO-bright $\mathrm{H}_{2}$ should be $\leq 5$ in our case. This is about a factor of 10 higher than model predictions, e.g., by Wolfire et al. (2010) and Smith et al. (2014). A possible cause of this difference is the assumed physical conditions; for example, the nominal cloud modeled in Wolfire et al. (2010) is relatively large, with a total column density of $1.5 \times 10^{22} \mathrm{~cm}^{-2}$ which is larger than the largest $N\left(\mathrm{H}_{\text {tot }}\right)$ that we found by a factor of 10 . They also assumed simplified geometries for the clouds. The applicability of their results to more translucent clouds 
of complicated geometries is not clear and theoretical investigations are needed. Another possibility to explain the large dark-gas fraction (compared with the $\mathrm{H}_{2}$ traced by $\mathrm{CO}$ ) and the scatter in the $W_{\mathrm{HI}}-N\left(\mathrm{H}_{\text {tot }}\right)$ relation, both seen in Figure 9, is the optical thickness of the H I 21-cm line (e.g., Fukui et al. 2014, 2015). Then, $W_{\mathrm{H} \text { I }}$ can be correlated with $N\left(\mathrm{H}_{\text {tot }}\right)$ as a function of the spin temperature $T_{\mathrm{s}}$ as

$$
W_{\mathrm{HI}}\left(\mathrm{K} \mathrm{km} \mathrm{s}^{-1}\right)=\left[T_{\mathrm{S}}(\mathrm{K})-T_{\mathrm{bg}}(\mathrm{K})\right] \cdot \Delta V_{\mathrm{HI}}\left(\mathrm{km} \mathrm{s}^{-1}\right) \cdot\left[1-\exp \left(-\tau_{\mathrm{H}}\right)\right],
$$

and

$$
\tau_{\mathrm{H}}=\frac{N_{\mathrm{H}_{\mathrm{tot}}}\left(\mathrm{cm}^{-2}\right)}{1.82 \times 10^{18}} \cdot \frac{1}{T_{\mathrm{S}}(\mathrm{K})} \cdot \frac{1}{\Delta V_{\mathrm{HI}}\left(\mathrm{km} \mathrm{s}^{-1}\right)},
$$

where $\Delta V_{\mathrm{H}_{\mathrm{I}}}$ is the $\mathrm{HI}$ line width [defined as $W_{\mathrm{HI}} /\left(\right.$ peak $\mathrm{HI}$ brightness temperature)], $T_{\mathrm{bg}}$ is the background continuum radiation temperature, and $\tau_{\mathrm{H}}$ is the $\mathrm{H}$ I optical depth. In Figure 9a, making an approximation by assuming that all of the gas is atomic and associated with MBM 53, 54, and 55 clouds, we overlay the model curves for several choices of $T_{\mathrm{s}}$ with $\Delta V_{\mathrm{HI}}=14 \mathrm{~km} \mathrm{~s}^{-1}$ (the median velocity dispersion in the vicinity of the MBM 53-55 complexes; see Fukui et al. 2014) and $T_{\mathrm{bg}}=2.7 \mathrm{~K}$ (the cosmic microwave background radiation). To illustrate the uncertainty of model curves due to the spread of $\Delta V_{\mathrm{HI}}$, we also plot the curves of $\Delta V_{\mathrm{HI}}=11$ and $22 \mathrm{~km} \mathrm{~s}^{-1}$ (which covers the half width of the $\Delta V_{\mathrm{H}}$ distribution of MBM 53-55) for $T_{\mathrm{s}}=20$ and $100 \mathrm{~K}$ as dotted lines. As inferred from the figure, the region with $T_{\mathrm{d}} \geq 20.5 \mathrm{~K}$ is almost optically thin. As the dust temperature decreases, H I becomes optically thicker with $T_{\mathrm{s}} \sim 100 \mathrm{~K}$ for $T_{\mathrm{d}}=19.5-20 \mathrm{~K}, T_{\mathrm{s}} \sim 60 \mathrm{~K}$ for $T_{\mathrm{d}}=18.5-19 \mathrm{~K}$, and $T_{\mathrm{s}} \sim 40 \mathrm{~K}$ for $T_{\mathrm{d}} \leq 18 \mathrm{~K}$.

We note that the current data considered in this study cannot distinguish between the two scenarios for the primary origin of the dark gas (CO-dark molecular gas, or optically thick atomic gas, or a mix of both contributions). Therefore systematic and large surveys of background radio sources for direct measurements of the H I optical depth are important (although such large surveys may not be feasible). We also look forward to the progress in theoretical work (e.g., detailed modeling of translucent clouds) for more detailed discussions of $\mathrm{CO}$-dark $\mathrm{H}_{2}$ hypothesis. The main achievement of this study is quantification of the distribution of $N\left(\mathrm{H}_{\mathrm{tot}}\right)$ and the dark gas by combining the Fermi-LAT $\gamma$-ray data and the Planck dust model. If we use the (uncorrected) $R$-based $N\left(\mathrm{H}_{\text {tot }}\right)$ and $\tau_{353}$-based $N\left(\mathrm{H}_{\text {tot }}\right)$ maps, the integral of $N\left(\mathrm{H}_{\text {tot }}\right)-N\left(\mathrm{HI}_{\text {thin }}\right)$ is 6.2 and 56.8 in units of $10^{22} \mathrm{~cm}^{-2} \mathrm{deg}^{2}$, respectively, whereas we obtained 16.5 in the same units through $\gamma$-ray data analysis. Then, the dark gas contribution (obtained by subtracting $2 X_{\mathrm{CO}} \int W_{\mathrm{CO}} d \Omega$ estimated to be $\sim 2.6$ ) based on uncorrected $R$-based and $\tau_{353}$-based $N\left(\mathrm{H}_{\text {tot }}\right)$ maps will be 3.6 and 54.2 , respectively, and is a factor of $\sim 4$ lower/higher than what we found $(16.5-2.6=13.9)$; therefore, the correction based on the $\gamma$-ray analysis is crucial. We also note that the required correction we found $\left(\sim 10 \%\right.$ increase of $N\left(\mathrm{H}_{\mathrm{tot}}\right)$ as $T_{\mathrm{d}}$ decreases by $1 \mathrm{~K}$ ) is the average of the studied region. A systematic study of other high-latitude regions by Fermi-LAT with the latest event selections and response functions (Pass 8) in combination with the Planck dust model and careful examinations of the properties of ISM tracers is required to examine the uniformity/variation of the ISM properties in the solar neighborhood. Investigating sub-regions of this study with more LAT data also would be worthwhile.

Finally we discuss the Hi emissivity spectrum obtained in this study summarized in Figure 10. To examine the systematic uncertainty, we repeated the same analysis in Section 4.3 [first searched for the coefficient of Equation (3) and then fit $\gamma$-ray data with narrow energy bins] using the IC model of pulsar-based CR source distribution and $z_{\mathrm{h}}=4 \mathrm{kpc}$, our baseline IC model but with areas of molecular clouds traced by CO masked, and our baseline IC model but with areas of IVCs masked (see Section 4.2). Another source of systematic uncertainty of the HI emissivity spectrum is the $W_{\mathrm{H} \text { I }}-$ to $-R$ ratio evaluated in Section 4.1. To examine this uncertainty, we divided the region with $T_{\mathrm{d}} \geq 20.5 \mathrm{~K}$ into six sub-regions, collectively spanning the region while requiring that the area of each sub-region has more than $10 \%$ of the whole area. The best-fit values of $W_{\mathrm{H}} / R$ of each sub-region were found to be within $+3.6 \% /-8.4 \%$ from the average. Although this uncertainty does not affect the slope of $T_{\mathrm{d}}$ dependence shown in Figure 4 , it changes the normalization of the emissivity spectrum independent of energy, and we added this uncertainty to that due to the modeling of $\gamma$-ray data (choice of the IC model and masking areas of clouds traced by CO or IVCs) as a linear sum. The obtained systematic uncertainty is shown by the shaded band in Figure 10. For comparison, we plotted the model curve for the LIS that we adopted and $\epsilon_{\mathrm{M}}$ of 1.84 in the same figure. To gauge the uncertainty in the emissivity model [mainly due to the uncertainty of the elemental composition of CRs and the cross sections other than proton-proton (p-p) collisions], we also plotted the model curve for $\epsilon_{\mathrm{M}}=1.45$ (the lowest value referred to in Mori 2009) which gives 15\%-20\% lower emissivity. We also plotted the emissivity spectrum of the local H i clouds (in different regions of the sky) measured by Abdo et al. (2009b) and Casandjian (2015) for comparison, in the analysis for which different LAT event selections and response functions were employed (the so-called Pass 6 and Pass 7 by Abdo et al. (2009b) and Casandjian (2015), respectively). Most recent studies of high-latitude regions by Fermi-LAT, e.g., local H I emissivities in Tibaldo et al. (2015) and H I emissivity of the Chamaeleon complex in Planck Collaboration (2015) find H I emissivity spectra similar 
to that of Casandjian (2015). One can recognize that our emissivity spectrum is apparently harder than the model curves and those from previous Fermi-LAT studies. However, within the systematic and statistical uncertainties the slope of our spectrum is consistent with those of the models (and previous Fermi-LAT results) above $1 \mathrm{GeV}$ in which the index of the emissivity spectrum follows that of CR protons (e.g., Aharonian \& Atoyan 2000). We thus do not claim nor deny a harder emissivity spectrum than that inferred from the CR spectra measured at the Earth. A more significant difference can be seen in the normalization. Our result agrees with the model with $\epsilon_{\mathrm{M}}=1.45$ except for the lowest energy bin, in which the degeneracy among model components are most severe due to the breadth of the point-spread function and for which the LIS is uncertain at the $\sim 20 \%$ level due to the solar modulation (e.g., see discussions by Abdo et al. 2009b). On the other hand, relevant studies by Fermi-LAT favor the model with $\epsilon_{\mathrm{M}}=1.85$. The difference is larger than the statistical and systematic uncertainties for energies below a few GeV. This difference cannot be fully explained by the uncertainty in the LAT effective area $(\sim 5 \%)^{15}$. It can be understood, at least partially, due to the assumption of the H I optical thickness. Abdo et al. (2009b) and Casandjian (2015) assumed a uniform $T_{\mathrm{s}}$ of $125 \mathrm{~K}$ and $140 \mathrm{~K}$, respectively, and Tibaldo et al. (2015) and Planck Collaboration (2015) assumed the optically thin case. If uniform $T_{\mathrm{s}}$ of such values (greater than or equal to $125 \mathrm{~K}$ ) is applied to our region, we will have smaller $N\left(\mathrm{H}_{\text {tot }}\right)$ on average than that we obtained in Figure 9, and larger H I emissivity.

More specifically, the difference comes from different assumptions on gas and dust properties. The method presented in this study is based on several assumptions: (A1) Optically-thin H I dominates the ISM gas in areas with high $T_{\mathrm{d}} .{ }^{16}$ (A2) $N\left(\mathrm{H}_{\text {tot }}\right) / R$ is constant for the same value of $T_{\mathrm{d}}$. We also assume that, through the $\gamma$-ray data analysis (Figure 4$)$, (A3) the $T_{\mathrm{d}}$-dependence of $N\left(\mathrm{H}_{\mathrm{tot}}\right) / R$ can be compensated for by employing the empirical function of Equation (3). On the other hand, (leaving aside small differences in the analysis procedure and detailed assumptions) a conventional template-fitting method (e.g., Abdo et al. 2010; Ackermann et al. 2011, 2012b; Casandjian 2015; Tibaldo et al. 2015; Planck Collaboration 2015) is based on the following assumptions on gas and dust properties: (B1) The ISM gas can be divided into the atomic gas, the molecular gas associated with CO emission, and the dark-gas. (B2) In each phase (the atomic, molecular and dark-gas), the gas and dust properties are uniform across the ROI (or sub-regions). More specifically, the statement (B2) can be broken down as follows. (B2a) Atomic hydrogen has uniform $T_{\mathrm{s}}$ and uniform $N(\mathrm{HI}) / D$, where $D$ is the employed dust map (e.g., $R$ and $\tau_{353}$ ). (B2b) Molecular hydrogen has uniform $X_{\mathrm{CO}}$ and uniform $N\left(\mathrm{H}_{2}\right) / D$. (B2c) Dark gas has uniform $N\left(\mathrm{H}_{\mathrm{DG}}\right) / D$ where $N\left(\mathrm{H}_{\mathrm{DG}}\right)$ is the column density of the dark gas. In short, the method presented here relies on the uniformity of $N\left(\mathrm{H}_{\mathrm{tot}}\right) / R$ for the same value of $T_{\mathrm{d}}$ regardless of the gas phases, while a conventional template-fitting method relies on the uniformity of $T_{\mathrm{s}}, X_{\mathrm{CO}}$, and $N(\mathrm{H}) / D$ in each gas phase across the ROI (or sub-regions). Therefore using a good dust tracer is crucial for the conventional method, and several alternative tracers of dust need to be compared to properly trace the ISM and CRs (e.g., Planck Collaboration 2015). The method developed here, on the other hand, has more flexibility to adjust $N\left(\mathrm{H}_{\text {tot }}\right) / D$. The obtained emissivity, however, depends on the calibration of the gas-to-dust ratio and the $\gamma$-ray fit in high $T_{\mathrm{d}}$ regions; therefore careful examination of the systematic uncertainty as we have done is required.

To discuss the difference from previous relevant studies by Fermi-LAT more quantitatively, we also employed a conventional template-fitting. As shown in Figure $2, R$ shows better correlation with $W_{\mathrm{H}}$ than $\tau_{353}$ does, and therefore we used the $R$ map as a dust tracer and performed a fit to $\gamma$-ray data. Details of the template preparation and obtained results are given in Appendix $\mathrm{D}$, in which the analysis using $N\left(\mathrm{H}_{\mathrm{tot} \text {,mod }}\right)$ with $T_{\mathrm{bk}}=20.5 \mathrm{~K}$ and $C=2$ [Equation (3)] is also shown for comparison. As shown by Figure D5, the two methods show similar data/model ratio, and their $\gamma$-ray model maps agree to within $\leq 5 \%$ with each other in most of the ROI. ${ }^{17}$ Nevertheless, the scaling factor for the atomic gas phase obtained by the conventional analysis is $0.924 \pm 0.022$, which agrees (within $\leq 10 \%$ ) with the model of $\epsilon=1.84$ like the other Fermi-LAT studies employing the similar method but is $\sim 35 \%$ larger than what we obtained using the $N\left(\mathrm{H}_{\text {tot,mod }}\right)$ map. A significant fraction of the difference is attributable to the inferred gas column density. As shown by Figure D6, while the two analyses show similar $N\left(\mathrm{H}_{\text {tot }}\right)$ in $T_{\mathrm{d}} \geq 19 \mathrm{~K}$, they give distinct $N\left(\mathrm{H}_{\text {tot }}\right)$ below $19 \mathrm{~K}$ where the conventional method has less flexibility to adjust $N\left(\mathrm{H}_{\text {tot }}\right)$. The difference is $\sim 12 \%$ in $T_{\mathrm{d}}=18.5-19 \mathrm{~K}$ and becomes gradually larger as $T_{\mathrm{d}}$ decreases; in $T_{\mathrm{d}}=17-17.5 \mathrm{~K}$, the difference is more than $25 \%$. We can also see that the conventional method requires significantly smaller IC emission as shown by Tables D4 and D5 and Figure D4, attributing more $\gamma$ rays to the gas-related component than our analysis does. As a result of these two effects, most of the difference of scale factors (H I emissivity) can be explained. Given the similarity of data/model ratios and $\gamma$-ray model maps, we do not rule out the conventional template-fitting analysis. However, the underlying

15 http://fermi.gsfc.nasa.gov/ssc/data/analysis/LAT_caveats.html

16 We recall that $T_{\mathrm{d}}$ was obtained under the assumption that the dust temperature is uniform.

17 Small differences are seen, e.g., in the upper-left corner $\left[(l, b) \sim\left(106^{\circ},-33^{\circ}\right)\right]$ where optically-thin $\mathrm{H}$ i dominates the ISM gas, the conventional method slightly overpredicts the data. 
assumption of the method, the uniformity of $N(\mathrm{H}) / D$ in each gas phase across the ROI, apparently does not agree with the result of the $\gamma$-ray analysis shown in Figure 4. [If the values of $X_{\mathrm{CO}}$ agree between the dust-fit and $\gamma$-ray analysis and $N\left(\mathrm{H}_{\mathrm{DG}}\right) / R$ is similar to $N\left(\mathrm{H}_{\mathrm{t}}\right.$ hin $) / R$, as inferred in Appendix $\mathrm{D}$, the conventional method predicts a uniform $N\left(\mathrm{H}_{\text {tot }}\right) / R$ independent of $T_{\mathrm{d}}$.] We therefore consider that the analysis we have developed is more accurate in this region of the sky, implying lower fraction of heavy CR nuclei in the solar neighborhood and/or smaller cross sections other than for $\mathrm{p}-\mathrm{p}$ collisions than previously inferred from gas emissivities in $\gamma$ rays. Finally we note that the conventional template-fitting method is well established and has long been applied for many previous Fermi-LAT studies, and has improved our understanding of the ISM and CRs.

Although our analysis was based on a few basic assumptions on gas and dust properties (optically-thin H I in regions with high $T_{\mathrm{d}}$ and a uniform $N\left(\mathrm{H}_{\mathrm{tot}}\right) / \mathrm{R}$ in each $T_{\mathrm{d}}$ range) and the plausible assumption that the $\gamma$-ray data can be used as a robust tracer of $N\left(\mathrm{H}_{\mathrm{tot}}\right)$, and we have carefully examined the systematic uncertainty, the method is still in an early phase of development and should be tested and improved by applying to other regions of the sky. In such future analyses, a comparison with the conventional method should be done at the same time to examine the merits and demerits of each method and the validity of the underlying assumptions, and to better understand the properties of the ISM and CRs in the solar neighborhood.

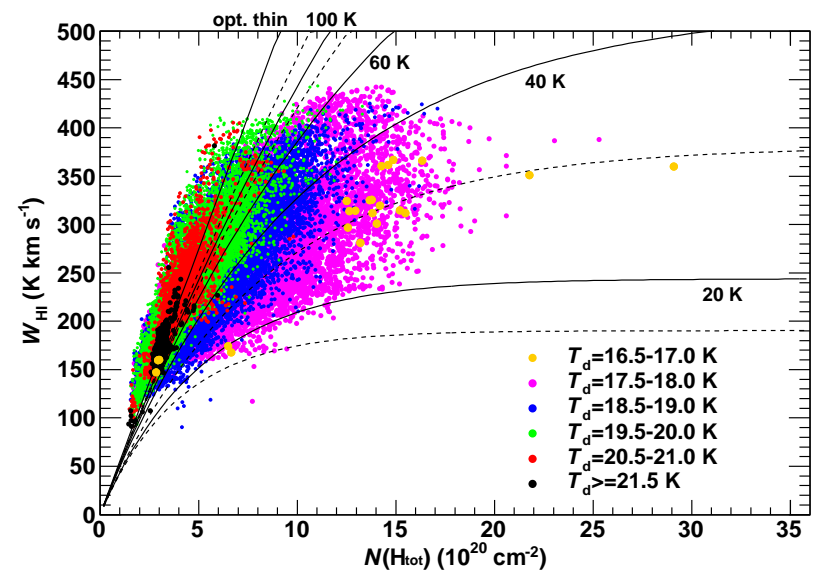

(a)

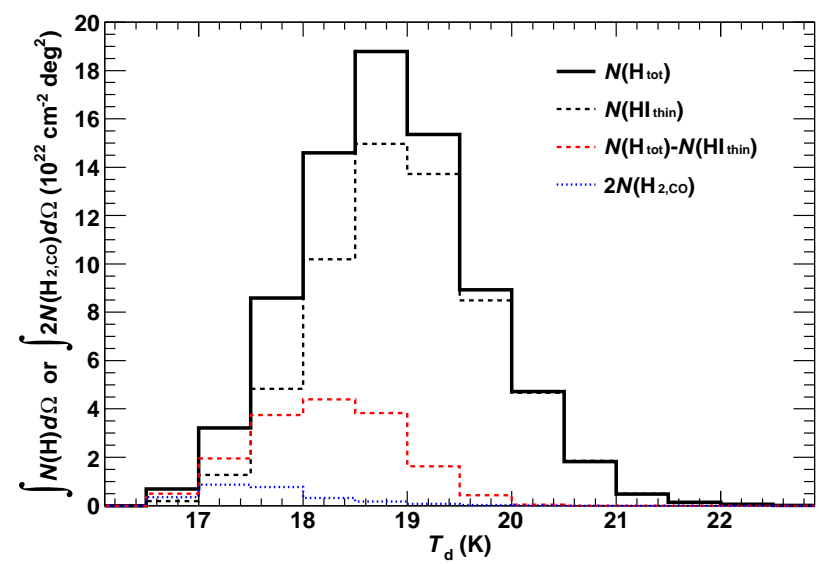

(b)

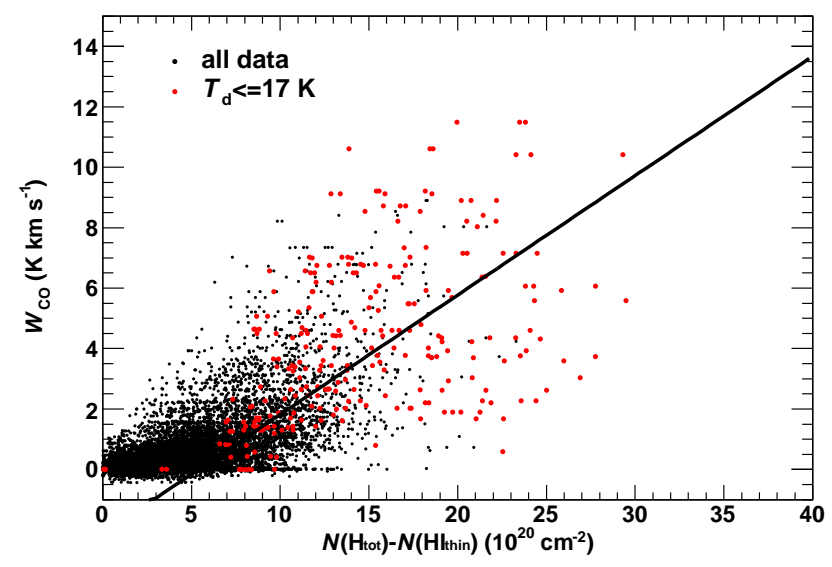

(c)

Figure 9. (a) The correlation between $W_{\mathrm{H} \text { I }}$ and the $N\left(\mathrm{H}_{\mathrm{tot}}\right)$ inferred by the $\gamma$-ray data analysis obtained from Equation (3) with $T_{\mathrm{bk}}=20.5 \mathrm{~K}$ and $C=2$. Making an approximation by assuming that all of the gas is atomic, the model curves for several choices of $T_{\mathrm{s}}$ are overlaid. Dotted lines illustrate the uncertainty of model curves for $T_{\mathrm{s}}=20$ and $100 \mathrm{~K}$. See the text for details. (b) The distribution of the integrated $\mathrm{H} \mathrm{I}$ column density for the optically thin case, $\int N\left(\mathrm{H} \mathrm{I}_{\mathrm{thin}}\right) d \Omega=1.82 \times 10^{18} \cdot \int W_{\mathrm{H}} d \Omega$, and that of the integrated excess gas column density above $N\left(\mathrm{HI}_{\mathrm{thin}}\right)$ calculated in $0.5-\mathrm{K}$ step $T_{\mathrm{d}}$ bins. The integrated $W_{\mathrm{CO}}$ as a measure of the CO-bright $\mathrm{H}_{2}$ is also plotted. (c) Scatter plot of $N\left(\mathrm{H}_{\text {tot }}\right)-N\left(\mathrm{HI}_{\mathrm{thin}}\right)$ vs. $W_{\mathrm{CO}}$ in the studied region. 


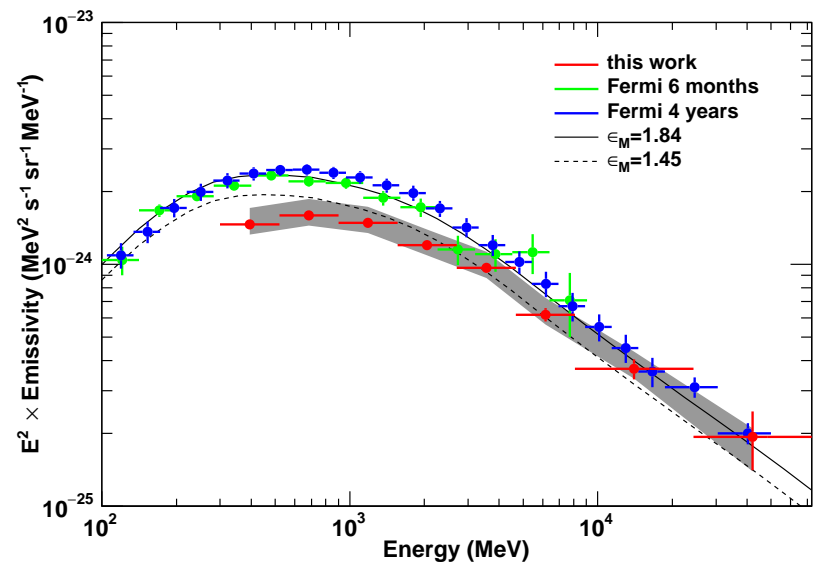

Figure 10. Summary of the H I emissivity spectrum obtained in this study compared with the model curves based on the LIS for $\epsilon_{\mathrm{M}}=1.45$ and 1.84, and the results of the relevant studies by Fermi-LAT, based on 6 months of observation and 4 years of observation by Abdo et al. (2009b) and Casandjian (2015), respectively. The shaded band shows the systematic uncertainty of our H I emissivity spectrum (see the text in Section 5 for details). 


\section{SUMMARY AND FUTURE PROSPECTS}

We carried out a study of the ISM and CRs using the Fermi-LAT data in the 0.3-72.9 GeV range and other interstellar gas tracers, in particular the Planck dust model, at Galactic longitudes from $60^{\circ}$ to $120^{\circ}$ and Galactic latitudes from $-60^{\circ}$ to $-28^{\circ}$. The region encompasses the nearby, high-Galactic latitude cloud complexes MBM 53, 54 , and 55 and a far-infrared loop-like structure in Pegasus. We found that neither $R$ nor $\tau_{353}$ estimated from Planck observations were good representations of the total gas column density. Instead, we found a systematic decrease of $N\left(\mathrm{H}_{\text {tot }}\right) / R$ or increase of $N\left(\mathrm{H}_{\text {tot }}\right) / \tau_{353}$ as dust temperature increases. We used the Fermi-LAT $\gamma$-ray data to quantify the total gas column density, obtaining the mass of the excess gas not traced by the H I 21-cm nor the CO 2.6-mm line surveys (dark gas) to be $\sim 25 \%$ of that from the H I gas in the optically thin case. The ratio of the mass of the excess gas to that of the $\mathrm{H}_{2}$ gas traced by $\mathrm{CO}$ is $\leq 5$. This ratio is about a factor of 10 larger than model predictions in the CO-dark $\mathrm{H}_{2}$ scenario, requiring a better modeling of the CO-dark $\mathrm{H}_{2}$ which is applicable to the translucent clouds studied here. Another possibility is that the HI has appreciable optical depth which implies a spin temperature of less than $100 \mathrm{~K}$ in regions with dust temperatures below $19 \mathrm{~K}$. The correlation of gas templates based on $\gamma$-ray data and dust temperature is crucial, since the dark gas contribution calculated from $R$ and $\tau_{353}$ without the correction is a factor of $\sim 4$ lower/higher than what we obtained. We measured the $\gamma$-ray emissivity spectrum and found it agrees with the model for the LIS with $\epsilon_{\mathrm{M}}=1.45$, while most of relevant Fermi-LAT studies based on different analysis methods and assumptions on ISM properties show 15\%-20\% higher emissivity normalizations in energies below a few $\mathrm{GeV}$. The difference can be understood as due to the different gas column density inferred in areas with low $T_{\mathrm{d}}$. Although we do not rule out the conventional template-fitting analysis, its underlying assumption does not coincide with the $T_{\mathrm{d}}$ dependence of $N\left(\mathrm{H}_{\mathrm{tot}}\right) / R$ we found; therefore we regard our analysis is more accurate in this region of the sky, implying lower fraction of heavy nuclei in local CRs and/or smaller cross sections other than for p-p interactions than previously inferred from gas emissivities in $\gamma$ rays. The method is still in early development phase, however, and needs to be tested and improved by applying to other high-latitude regions. In such studies, a comparison with the conventional template-fitting should also be carried out to examine the limitations of each method and to better understand the properties of the ISM and CRs in the solar neighborhood.

The Fermi LAT Collaboration acknowledges generous ongoing support from a number of agencies and institutes that have supported both the development and the operation of the LAT as well as scientific data analysis. These include the National Aeronautics and Space Administration and the Department of Energy in the United States, the Commissariat à l'Energie Atomique and the Centre National de la Recherche Scientifique / Institut National de Physique Nucléaire et de Physique des Particules in France, the Agenzia Spaziale Italiana and the Istituto Nazionale di Fisica Nucleare in Italy, the Ministry of Education, Culture, Sports, Science and Technology (MEXT), High Energy Accelerator Research Organization (KEK) and Japan Aerospace Exploration Agency (JAXA) in Japan, and the K. A. Wallenberg Foundation, the Swedish Research Council and the Swedish National Space Board in Sweden. Additional support for science analysis during the operations phase is gratefully acknowledged from the Istituto Nazionale di Astrofisica in Italy and the Centre National d'Études Spatiales in France.

We thank T. M. Dame for providing the moment-masked CO data. Some of the results in this paper have been derived using the HEALPix (Górski et al. 2005) package.

Facilities: Fermi (LAT), Planck

Software: Fermi Science Tools, GALPROP, HEALPix, ROOT 


\section{APPENDIX}

\section{A. TREATMENT OF THE INFRARED SOURCES}

In the Planck dust model maps we identified several regions with high $T_{\mathrm{d}}$, indicating localized heating by stars. We refilled these areas in the $R, \tau_{353}$, and $T_{\mathrm{d}}$ maps with the average of the peripheral pixels (since we used HEALPix maps of order 9 , the pixel size is $\sim 0.013 \mathrm{deg}^{2}$ ): values in a circular region with radius of $r_{1}$ are filled with the average of pixels in a ring with inner radius of $r_{1}$ and outer radius of $r_{2}$. For each region, the central position $(l, b), r_{1}$, and $r_{2}$ are summarized in Table A1. We used a large radius for the region of high $T_{\mathrm{d}}$ located near $3 \mathrm{C} 454.3$, even though the origin of such high temperature is unknown. Because 3C 454.3 is a very bright $\gamma$-ray source (see Figure 8), the impact on the $\gamma$-ray data analysis was small.

Table A1. Infrared sources excised and interpolated across in the Planck dust maps

\begin{tabular}{ccccc}
\hline \hline \multicolumn{2}{c}{ Position } & $r_{1}$ & $r_{2}$ & Object name \\
\cline { 1 - 3 }$l(\mathrm{deg})$ & $b(\mathrm{deg})$ & $(\mathrm{deg})$ & $(\mathrm{deg})$ & \\
\cline { 1 - 3 } 79.61 & -30.25 & 0.12 & 0.15 & \\
82.85 & -50.65 & 0.12 & 0.15 & \\
83.10 & -45.46 & 0.12 & 0.15 & \\
86.30 & -38.20 & 0.60 & 0.65 & 3C 454.3 (Active galactic nucleus) \\
87.46 & -29.73 & 0.12 & 0.15 & NGC 7339 (Radio galaxy) \\
87.57 & -39.12 & 0.12 & 0.15 & \\
93.53 & -40.35 & 0.12 & 0.15 & RAFGL 3068 (Variable star) \\
93.91 & -40.47 & 0.12 & 0.15 & NGC 7625 (Interacting galaxies) \\
97.29 & -32.52 & 0.12 & 0.15 & IC 5298 (Seyfert2 galaxy) \\
98.88 & -36.55 & 0.12 & 0.15 & NGC 7678 (Active galactic nucleus) \\
104.26 & -40.58 & 0.12 & 0.15 & \\
104.46 & -40.14 & 0.12 & 0.15 & \\
111.37 & -36.00 & 0.12 & 0.15 & \\
\hline
\end{tabular}




\section{B. INTERMEDIATE-VELOCITY CLOUDS}

In our ROI, we have identified coherent structures of atomic hydrogen with velocities from -80 to $-30 \mathrm{~km} \mathrm{~s} \mathrm{shich}^{-1}$ correspond to some of the IVCs in the southern sky (Wakker 2001), while the main H I clouds have velocities from -30 to $+20 \mathrm{~km} \mathrm{~s}^{-1}$, as shown by Figure B1. In panel (b) of the figure, we can identify a strip-like structure which has an intensity peak at $(l, b) \sim\left(87^{\circ},-38^{\circ}\right)$ and runs toward the lower-left corner of the image. We can also identify intense emissions in the upper-left corner of the ROI, although some fraction of them are likely to be the contamination from the Galactic plane, not the IVCs. In Section 4.2, we masked those structures [using green lines in panel (b)] to examine the effect on the $\gamma$-ray data analysis.

The relative contribution of the clouds to the $\gamma$-ray flux (assuming uniform CR density) and the mass of the ISM gas (assuming the same distance) can be evaluated by integrating $W_{\mathrm{H}_{\mathrm{I}}}$ in the ROI. The relative contribution of the main clouds (defined as the H I clouds having velocities from -30 to $+20 \mathrm{~km} \mathrm{~s}^{-1}$ ) to the whole emission of $W_{\mathrm{H} \text { I }}$ (integrated from -450 to $+400 \mathrm{~km} \mathrm{~s}^{-1}$ ) was found to be $\sim 93.3 \%$. The contribution of IVCs were evaluated by integrating $W_{\mathrm{H} \text { I }}$ having velocities from -80 to $-30 \mathrm{~km} \mathrm{~s}^{-1}$ over the entire ROI, and were found to be $\sim 5.5 \%$.

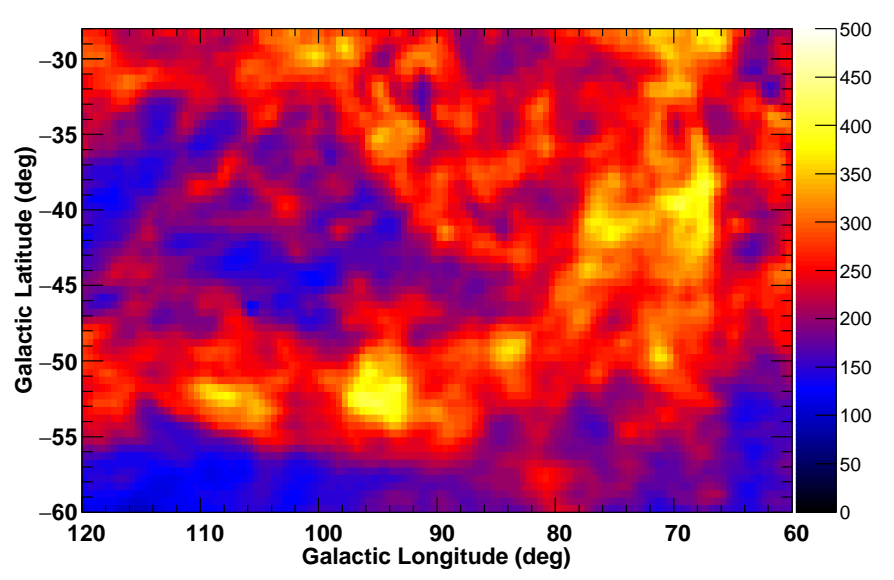

(a)

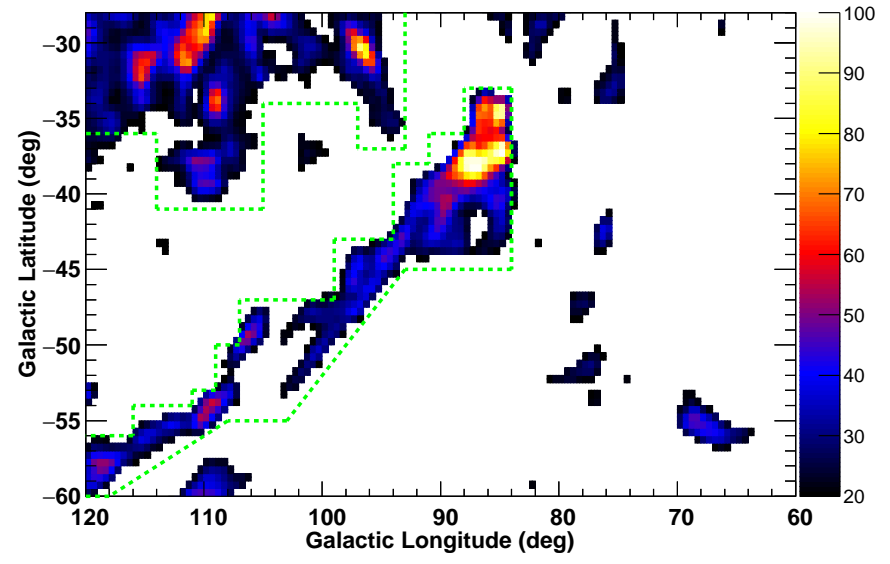

(b)

Figure B1. The $W_{\mathrm{H}_{\mathrm{I}}}$ map in the velocities (a) from -30 to $+20 \mathrm{~km} \mathrm{~s}^{-1}$ and (b) from -80 to $-30 \mathrm{~km} \mathrm{~s}^{-1}$. The former shows the structure of the main clouds, while the latter shows the distribution of IVCs. The dotted lines in panel (b) indicate the areas to be masked in Section 4.2. 


\section{RESULTS WITH THE MAPS SORTED BY DUST TEMPERATURE}

We split the $N\left(\mathrm{H}_{\text {tot }}\right)$ template map (constructed from $R$ or $\tau_{353}$ ) into four based on $T_{\mathrm{d}}$, with $T_{\mathrm{d}} \leq 18 \mathrm{~K}, T_{\mathrm{d}}=18-19 \mathrm{~K}$, $T_{\mathrm{d}}=19-20 \mathrm{~K}$ and $T_{\mathrm{d}} \geq 20 \mathrm{~K}$, and fit the $\gamma$-ray data with Equation (1) using the four template maps, with scaling factors ( $c_{1}$ for each of the four templates) free to individually vary instead of using a single $N\left(\mathrm{H}_{\text {tot }}\right)$ map. The obtained fit parameters and the spectrum of each component are summarized in Tables C2 and C3 and Figure C2.

Table C2. Results with the $R$-based $N\left(\mathrm{H}_{\text {tot }}\right)$ maps sorted by $T_{d}$

\begin{tabular}{|c|c|c|c|c|c|c|c|c|}
\hline $\begin{array}{l}\text { Energy } \\
(\mathrm{GeV})\end{array}$ & $\begin{array}{c}c_{1,1} \\
\left(T_{\mathrm{d}} \leq 18 \mathrm{~K}\right)\end{array}$ & $\begin{array}{c}c_{1,2} \\
(18-19 \mathrm{~K})\end{array}$ & $\begin{array}{c}c_{1,3} \\
(19-20 \mathrm{~K})\end{array}$ & $\begin{array}{c}c_{1,4} \\
\left(T_{\mathrm{d}} \geq 20 \mathrm{~K}\right)\end{array}$ & $c_{2 \mathrm{n}}$ & $c_{2 \mathrm{i}}$ & $\begin{array}{c}I_{\text {iso }} \\
\left(\text { norm }^{\mathrm{a}}\right)\end{array}$ & $\begin{array}{c}I_{\text {iso }} \\
\text { (index) }\end{array}$ \\
\hline $0.3-0.9$ & $0.84 \pm 0.02$ & $0.80 \pm 0.03$ & $0.75 \pm 0.04$ & $0.70 \pm 0.06$ & $0.70 \pm 0.18$ & $0.00 \pm 0.16$ & $3.94 \pm 0.06$ & $2.22 \pm 0.02$ \\
\hline $0.9-2.7$ & $0.91 \pm 0.02$ & $0.81 \pm 0.03$ & $0.78 \pm 0.04$ & $0.63 \pm 0.05$ & $0.84 \pm 0.17$ & $0.39 \pm 0.25$ & $0.89 \pm 0.03$ & $2.36 \pm 0.05$ \\
\hline $2.7-8.1$ & $1.00 \pm 0.05$ & $0.89 \pm 0.06$ & $0.76 \pm 0.07$ & $0.73 \pm 0.10$ & $1.17 \pm 0.27$ & $-1.0^{\mathrm{b}}$ & $0.17 \pm 0.01$ & $2.64 \pm 0.11$ \\
\hline $8.1-72.9$ & $0.84 \pm 0.14$ & $0.54 \pm 0.18$ & $0.40 \pm 0.23$ & $0.16 \pm 0.32$ & $1.54 \pm 0.56$ & $-0.44 \pm 0.21$ & $0.05 \pm 0.01$ & $2.69 \pm 0.10$ \\
\hline
\end{tabular}

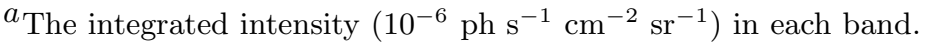

${ }^{b}$ Not well determined and reached at the smallest parameter boundary we set.

Note - The errors are 1-sigma statistical uncertainties. Each of the four scale factors $\left(c_{1,1}, c_{1,2}, c_{1,3}\right.$, and $\left.c_{1,4}\right)$ gives the normalization for a specified range of $T_{\mathrm{d}}$ of the gas-related component in each energy bin. The scale factor for IC $\left(c_{2}\right)$ is modeled by a power law in each energy bin (from $E_{\min }$ to $E_{\max }$ ) as $c_{2}(E)=c_{2 \mathrm{n}} \cdot\left(E / E_{0}\right)^{c_{2 \mathrm{i}}}$ where $E_{0}=\sqrt{E_{\min } \cdot E_{\mathrm{max}}} \cdot I_{\text {iso }}$ is modeled with a power law with the integrated intensity and the photon index as free parameters.

Table C3. Results with the $\tau_{353}$-based $N\left(\mathrm{H}_{\text {tot }}\right)$ maps sorted by $T_{d}$

\begin{tabular}{|c|c|c|c|c|c|c|c|c|}
\hline $\begin{array}{l}\text { Energy } \\
(\mathrm{GeV})\end{array}$ & $\begin{array}{c}c_{1,1} \\
\left(T_{\mathrm{d}} \leq 18 \mathrm{~K}\right)\end{array}$ & $\begin{array}{c}c_{1,2} \\
(18-19 \mathrm{~K})\end{array}$ & $\begin{array}{c}c_{1,3} \\
(19-20 \mathrm{~K})\end{array}$ & $\begin{array}{c}c_{1,4} \\
\left(T_{\mathrm{d}} \geq 20 \mathrm{~K}\right)\end{array}$ & $c_{2 \mathrm{n}}$ & $c_{2 \mathrm{i}}$ & $\begin{array}{c}I_{\text {iso }} \\
\left(\text { norm }^{\mathrm{a}}\right)\end{array}$ & $\begin{array}{c}I_{\text {iso }} \\
\text { (index) }\end{array}$ \\
\hline $0.3-0.9$ & $0.39 \pm 0.01$ & $0.47 \pm 0.01$ & $0.53 \pm 0.02$ & $0.63 \pm 0.03$ & $0.28 \pm 0.14$ & $0.07 \pm 0.42$ & $4.06 \pm 0.07$ & $2.22 \pm 0.02$ \\
\hline $0.9-2.7$ & $0.41 \pm 0.01$ & $0.45 \pm 0.02$ & $0.52 \pm 0.02$ & $0.54 \pm 0.04$ & $0.59 \pm 0.18$ & $0.44 \pm 0.32$ & $0.91 \pm 0.03$ & $2.37 \pm 0.04$ \\
\hline $2.7-8.1$ & $0.45 \pm 0.02$ & $0.50 \pm 0.03$ & $0.50 \pm 0.05$ & $0.60 \pm 0.08$ & $1.04 \pm 0.28$ & $-1.0^{\mathrm{b}}$ & $0.17 \pm 0.01$ & $2.58 \pm 0.11$ \\
\hline $8.1-72.9$ & $0.39 \pm 0.06$ & $0.32 \pm 0.10$ & $0.30 \pm 0.14$ & $0.19 \pm 0.25$ & $1.41 \pm 0.58$ & $-0.47 \pm 0.23$ & $0.05 \pm 0.01$ & $2.67 \pm 0.10$ \\
\hline
\end{tabular}

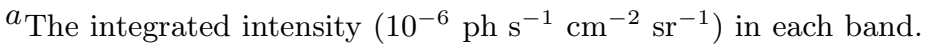

${ }^{b}$ Not well determined and reached at the smallest parameter boundary we set.

Note - The errors are 1-sigma statistical uncertainties. Each of the four scale factors $\left(c_{1,1}, c_{1,2}, c_{1,3}\right.$, and $\left.c_{1,4}\right)$ gives the normalization for a specified range of $T_{\mathrm{d}}$ of the gas-related component in each energy bin. The scale factor for IC ( $\left.c_{2}\right)$ is modeled by a power law in each energy bin (from $E_{\min }$ to $E_{\max }$ ) as $c_{2}(E)=c_{2 \mathrm{n}} \cdot\left(E / E_{0}\right)^{c_{2 \mathrm{i}}}$ where $E_{0}=\sqrt{E_{\min } \cdot E_{\max }}$. $I_{\text {iso }}$ is modeled with a power law with the integrated intensity and the photon index as free parameters. 


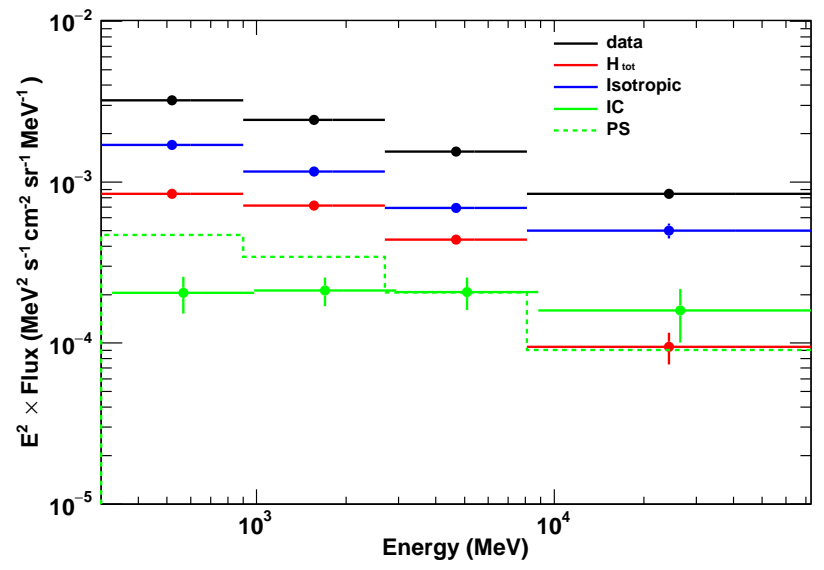

(a)

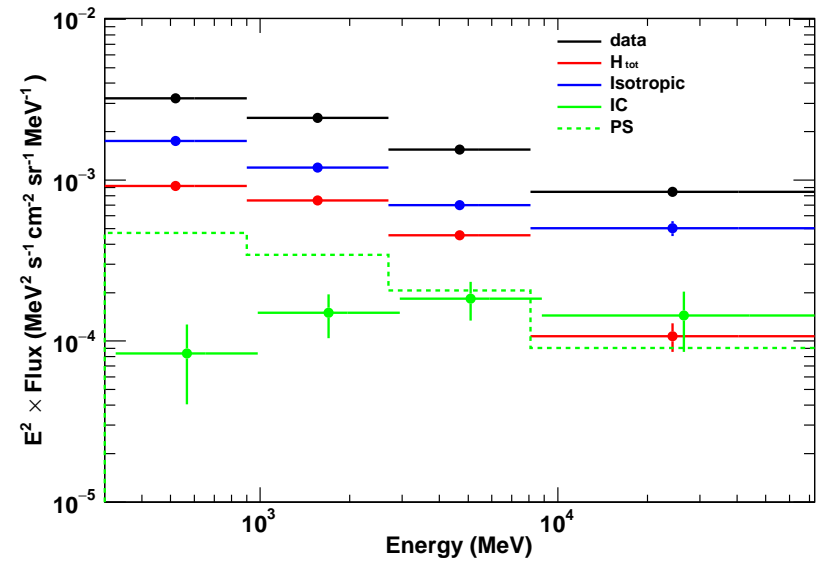

(b)

Figure C2. Spectrum of each component obtained by the four $N\left(\mathrm{H}_{\text {tot }}\right)$ maps sorted by $T_{\mathrm{d}}$, for (a) $R$-based analysis and (b) $\tau_{353}$-based analysis. 


\section{RESULTS BY A CONVENTIONAL TEMPLATE-FITTING METHOD}

In order to prepare a template map of the dark gas, we fit the original $R$ map with a linear combination of $N(\mathrm{H}$ I) map (Figure 1a) and the $W_{\mathrm{CO}}$ map (Figure $\left.1 \mathrm{~b}\right)$ : for simplicity we assumed the optically thin case to construct $N(\mathrm{Hi})$ map. Then, the fit to the $R$ map is expressed as

$$
R(l, b)=y_{\mathrm{H} \text { I }} \cdot N\left(\mathrm{HI}_{\mathrm{thin}}\right)(l, b)+y_{\mathrm{CO}} \cdot W_{\mathrm{CO}}(l, b),
$$

where $y_{\mathrm{H}}$ and $y_{\mathrm{CO}}$ are coefficients for $N(\mathrm{HI})$ and $W_{\mathrm{CO}}$ maps, respectively. Through least-squares fitting, we obtained $y_{\mathrm{H}}=(2.787 \pm 0.008) \times 10^{-28} \mathrm{~W} \mathrm{~m}^{-2} \mathrm{sr}^{-1} \mathrm{~cm}^{2}$ and $y_{\mathrm{CO}}=(5.41 \pm 0.13) \times 10^{-8} \mathrm{~W} \mathrm{~m}^{-2} \mathrm{sr}^{-1}\left(\mathrm{~K} \mathrm{~km} \mathrm{~s}^{-1}\right)^{-1} \cdot \mathrm{We} \mathrm{can}$ convert $y_{\mathrm{H}_{\mathrm{I}}}$ into the ratio of $N\left(\mathrm{HI}_{\mathrm{thin}}\right)$ to $R$ as $(0.3588 \pm 0.0010) \times 10^{28} \mathrm{~cm}^{-2}\left(\mathrm{~W} \mathrm{~m}^{-2} \mathrm{sr}^{-1}\right)^{-1}$, and calculate the dust-fit based $X_{\mathrm{CO}}$ from $y_{\mathrm{H} \text { I }}$ and $y_{\mathrm{CO}}$ as $X_{\mathrm{CO} \text {,dust }}=y_{\mathrm{CO}} / 2 y_{\mathrm{H}_{\mathrm{I}}}=(0.974 \pm 0.023) \times 10^{21} \mathrm{~cm}^{-2}\left(\mathrm{~K} \mathrm{~km} \mathrm{~s}^{-1}\right)^{-1}$. We used the positive residuals as a template map for dark gas $\left(R_{\mathrm{res}}^{\mathrm{DG}}\right)$ as shown in Figure D3. Then, instead of Equation $(1)$, the $\gamma$-ray intensities can be modeled as

$$
\begin{aligned}
I_{\gamma}(l, b, E)= & {\left[c_{\mathrm{HI}}(E) \cdot N\left(\mathrm{HI}_{\mathrm{thin}}\right)(l, b)+c_{\mathrm{CO}}(E) \cdot W_{\mathrm{CO}}(l, b)+c_{\mathrm{DG}}(E) \cdot R_{\mathrm{res}}^{\mathrm{DG}}(l, b)\right] q_{\gamma}(E) } \\
& +c_{2}(E) \cdot I_{\mathrm{IC}}(l, b, E)+I_{\mathrm{iso}}(E)+\sum_{j} \operatorname{PS}_{j}(l, b, E),
\end{aligned}
$$

where $c_{\mathrm{HI}}(E), c_{\mathrm{CO}}(E)$ and $c_{\mathrm{DG}}(E)$ are scale factors for the atomic gas, the molecular gas traced by CO, and the dark gas, respectively. Since we employed three template gas maps instead of a single $N\left(\mathrm{H}_{\text {tot }}\right)$ map (and therefore we have more free parameters), we used wider energy ranges as we did in Section 4.2. We note that this analysis was simple and did not adopt detailed procedures such as a denoising of $R_{\text {res }}^{\mathrm{DG}}$ and iterative fittings in dust and $\gamma$-rays employed by, e.g., Planck Collaboration (2015). In this analysis we do not aim to perform an optimized analysis in the framework of the conventional template-fitting technique, but to compare the method we have developed in this paper with a conventional one semiquantitatively.

The obtained best-fit parameters are summarized in Table D4. For comparison, we also tabulate the best-fit parameters obtained by using the $N\left(\mathrm{H}_{\mathrm{tot}, \mathrm{mod}}\right)$ map with $T_{\mathrm{bk}}=20.5 \mathrm{~K}$ and $C=2$ [see Equation (3) in Section 4.3 ] in Table D5. The average of $c_{\mathrm{H}}, c_{\mathrm{CO}}$, and $c_{\mathrm{DG}}$ are $0.924 \pm 0.022,(1.658 \pm 0.070) \times 10^{20} \mathrm{~cm}^{-2}\left(\mathrm{~K} \mathrm{~km} \mathrm{~s}^{-1}\right)^{-1}$, and $(0.360 \pm 0.011) \times 10^{28} \mathrm{~cm}^{-2}\left(\mathrm{~W} \mathrm{~m}^{-2} \mathrm{sr}^{-1}\right)^{-1}$, respectively. (For comparison, the average of $c_{1}$ obtained by the analysis using the $N\left(\mathrm{H}_{\text {tot,mod }}\right)$ map is $0.677 \pm 0.009$.) From these scale factors, we can calculate $X_{\mathrm{CO}}$ based on $\gamma$-ray data analysis as $X_{\mathrm{CO}, \gamma}=(0.897 \pm 0.043) \times 10^{20} \mathrm{~cm}^{-2}\left(\mathrm{~K} \mathrm{~km} \mathrm{~s}^{-1}\right)^{-1}$, and the conversion factor from $R$ to the dark gas column density, $X_{\mathrm{DG}}$ as $c_{\mathrm{DG}} / c_{\mathrm{H} \text { I }}=(0.3911 \pm 0.0093) \times 10^{28} \mathrm{~cm}^{-2}\left(\mathrm{~W} \mathrm{~m}^{-2} \mathrm{sr}^{-1}\right)^{-1}$. They agree with the corresponding quantities obtained from the dust fit $\left(X_{\mathrm{CO} \text {, dust }}\right.$ and $1 / y_{\mathrm{H}}$, respectively) described above within $\leq 10 \%$.

The values of $\ln L$ obtained by the conventional template-fitting method and the analysis using the single $N\left(\mathrm{H}_{\mathrm{tot}}\right.$,mod $)$ map summed over individual energy ranges in $0.3-72.9 \mathrm{GeV}$ are 1262809.3 and 1262815.2 , respectively. ${ }^{18}$ The spectra of each component from the two analyses are summarized in Figure D4 and fit residuals are compared in Figure D5, in which the ratio of the $\gamma$-ray model maps is also presented. We also show in Figure D6 the integrated gas column densities of each phase (dotted histograms) and the integrated total gas column density (thick solid histogram) as a function of $T_{\mathrm{d}}$. The integrals of $N\left(\mathrm{HI}_{\mathrm{thin}}\right), N\left(\mathrm{H}_{\mathrm{DG}}\right)$, and $2 X_{\mathrm{CO}} W_{\mathrm{CO}}$ are $60.9,7.4$ and $1.9 \mathrm{in}$ units of $10^{22} \mathrm{~cm}^{-2} \mathrm{deg}{ }^{2}$, respectively, where $10^{22} \mathrm{~cm}^{-2} \mathrm{deg}^{2}$ corresponds to $\sim 740 \mathrm{M}_{\odot}$ for $d=150 \mathrm{pc}$ (see Section 5 for details). In the same plot, the integral of $N\left(\mathrm{H}_{\text {tot,mod }}\right)$ (already shown in Figure $9 \mathrm{~b}$ ) is also presented for comparing the inferred total gas column density distributions between the two analyses.

18 We note that we give the values of $\ln L$ for reference. Since our conventional template-fitting analysis is not optimized as described in the text, a statistical comparison based on the values of $\ln L$ is not appropriate. 
Table D4. Best-fit parameters with 1-sigma statistical uncertainties, obtained by the conventional template-fitting method

\begin{tabular}{cccccccc}
\hline \hline $\begin{array}{c}\text { Energy } \\
(\mathrm{GeV})\end{array}$ & $c_{\mathrm{H} \mathrm{I}}$ & $c_{\mathrm{CO}}{ }^{\mathrm{a}}$ & $c_{\mathrm{DG}}{ }^{\mathrm{b}}$ & $c_{2 \mathrm{n}}{ }^{\mathrm{c}}$ & $c_{2 \mathrm{i}}{ }^{\mathrm{c}}$ & $\begin{array}{c}I_{\text {iso }} \\
\left(\mathrm{norm}^{\mathrm{d}}\right)\end{array}$ & $\begin{array}{c}I_{\text {iso }} \\
(\text { index })\end{array}$ \\
\hline $0.3-0.9$ & $0.92 \pm 0.03$ & $1.57 \pm 0.10$ & $0.34 \pm 0.02$ & $0.35 \pm 0.18$ & $0.06 \pm 0.21$ & $3.72 \pm 0.07$ & $2.24 \pm 0.02$ \\
$0.9-2.7$ & $0.93 \pm 0.04$ & $1.62 \pm 0.12$ & $0.39 \pm 0.02$ & $0.33 \pm 0.12$ & $0.53 \pm 0.48$ & $0.85 \pm 0.03$ & $2.38 \pm 0.04$ \\
$2.7-8.1$ & $1.03 \pm 0.09$ & $2.54 \pm 0.27$ & $0.36 \pm 0.04$ & $0.13 \pm 0.06$ & $3.51 \pm 0.70$ & $0.17 \pm 0.01$ & $2.01 \pm 0.08$ \\
$8.1-72.9$ & $0.56 \pm 0.25$ & $1.86 \pm 0.69$ & $0.46 \pm 0.11$ & $0.89 \pm 0.45$ & $0.63 \pm 0.30$ & $0.06 \pm 0.01$ & $2.61 \pm 0.09$ \\
\hline
\end{tabular}

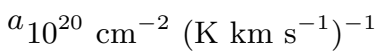

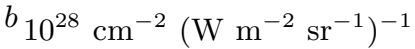

${ }^{c}$ Since the IC model is not a structured component and its intensity is lower than that of the isotropic component, and the energy bands analyzed are relatively narrow, the obtained values for the normalization and index $\left(c_{2 \mathrm{n}}\right.$ and $\left.c_{2 \mathrm{i}}\right)$ are uncertain and quite correlated (e.g., smaller normalization and larger index in 2.7-8.1 GeV than those in other energy ranges).

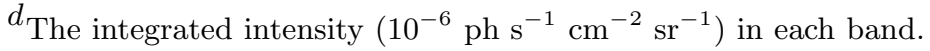

Note - In each energy bin, $c_{\mathrm{H}}(E), c_{\mathrm{CO}}(E)$ and $c_{\mathrm{DG}}(E)$ give scale factors for the atomic gas, the molecular gas, and the dark gas, respectively. The scale factor for IC $\left(c_{2}\right)$ is modeled by a power law in each energy bin (from $E_{\text {min }}$ to $\left.E_{\text {max }}\right)$ as $c_{2}(E)=c_{2 \mathrm{n}} \cdot\left(E / E_{0}\right)^{c_{2 \mathrm{i}}}$ where $E_{0}=\sqrt{E_{\min } \cdot E_{\max }} \cdot I_{\text {iso }}$ is modeled with a power law with the integrated intensity and the photon index as free parameters.

Table D5. Best-fit parameters with 1-sigma statistical uncertainties, obtained by the fit using the $N\left(\mathrm{H}_{\text {tot,mod }}\right)$ map

\begin{tabular}{cccccc}
\hline \hline $\begin{array}{c}\text { Energy } \\
(\mathrm{GeV})\end{array}$ & $c_{1}$ & $c_{2 \mathrm{n}^{\mathrm{a}}}$ & $c_{2 \mathrm{i}}{ }^{\mathrm{a}}$ & $\begin{array}{c}I_{\text {iso }} \\
\left(\mathrm{norm}^{\mathrm{b}}\right)\end{array}$ & $\begin{array}{c}I_{\text {iso }} \\
(\text { index })\end{array}$ \\
\hline $0.3-0.9$ & $0.65 \pm 0.01$ & $0.85 \pm 0.15$ & $-0.02 \pm 0.12$ & $3.92 \pm 0.08$ & $2.22 \pm 0.02$ \\
$0.9-2.7$ & $0.70 \pm 0.01$ & $0.77 \pm 0.11$ & $0.40 \pm 0.26$ & $0.89 \pm 0.02$ & $2.36 \pm 0.05$ \\
$2.7-8.1$ & $0.77 \pm 0.03$ & $0.45 \pm 0.21$ & $2.06 \pm 0.66$ & $0.18 \pm 0.01$ & $1.98 \pm 0.08$ \\
$8.1-72.9$ & $0.64 \pm 0.09$ & $0.97 \pm 0.43$ & $-0.60 \pm 0.28$ & $0.06 \pm 0.01$ & $2.61 \pm 0.09$ \\
\hline
\end{tabular}

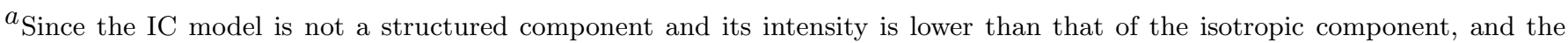
energy bands analyzed are relatively narrow, the obtained values for the normalization and index $\left(c_{2 \mathrm{n}}\right.$ and $\left.c_{2 \mathrm{i}}\right)$ are uncertain and quite correlated (e.g., smaller normalization and larger index in 2.7-8.1 GeV than those in other energy ranges).

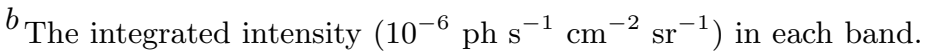

Note - In each energy bin, $c_{1}$ gives the scale factor of the gas-related component. The scale factor for IC $\left(c_{2}\right)$ is modeled by a power law in each energy bin (from $E_{\min }$ to $E_{\max }$ ) as $c_{2}(E)=c_{2 \mathrm{n}} \cdot\left(E / E_{0}\right)^{c_{2 i}}$ where $E_{0}=\sqrt{E_{\min } \cdot E_{\max }}$. $I_{\text {iso }}$ is modeled with a power law with the integrated intensity and the photon index as free parameters. 


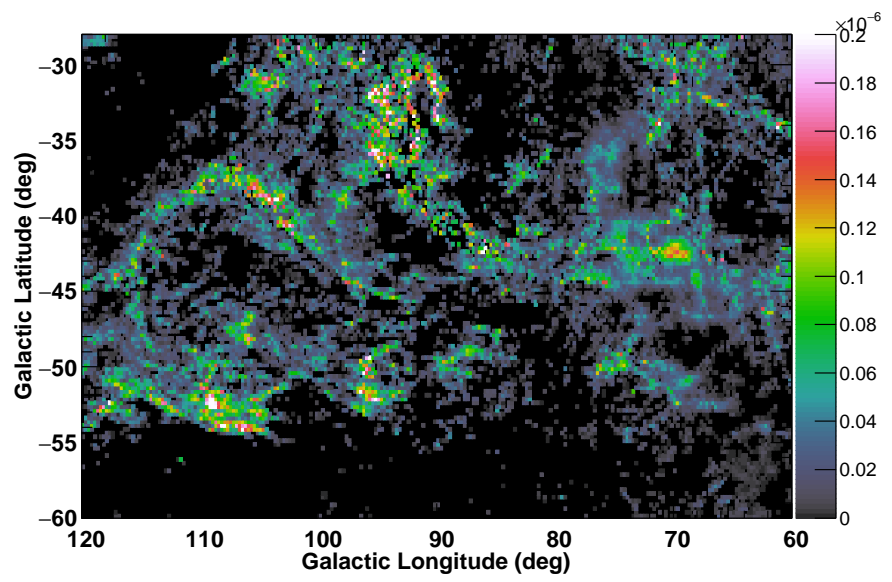

Figure D3. The template dark gas map based on the Planck $R$ map in units of $\mathrm{W} \mathrm{m}^{-2} \mathrm{sr}^{-1}$.

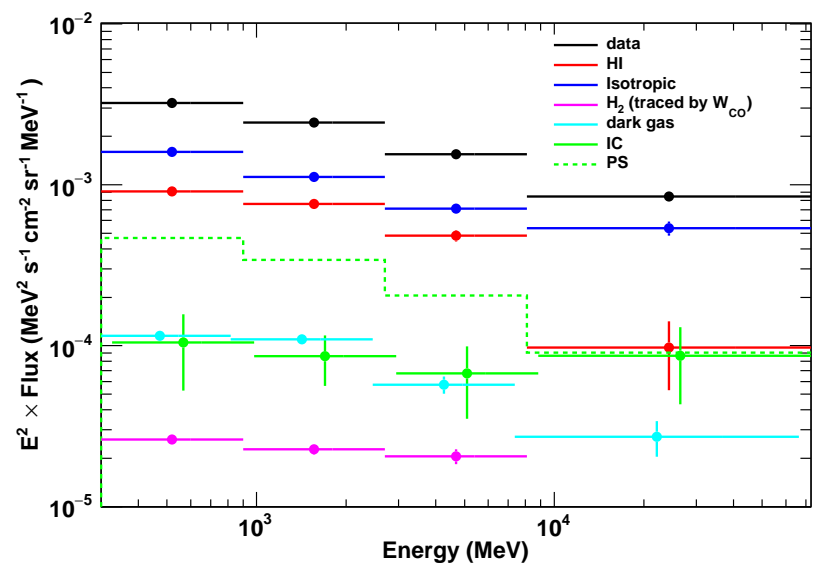

(a)

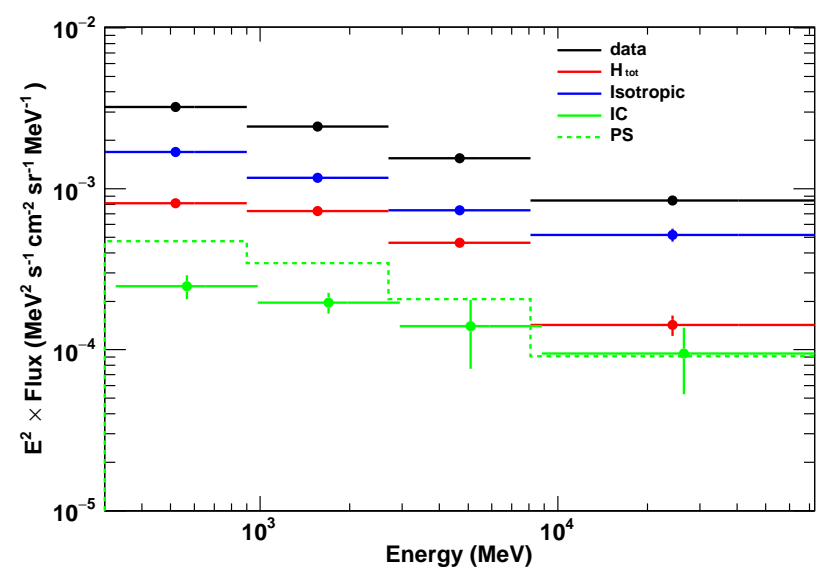

(b)

Figure D4. Spectrum of each component obtained by (a) the conventional template-fitting method and (b) the analysis using the $N\left(\mathrm{H}_{\text {tot,mod }}\right)$ map. 


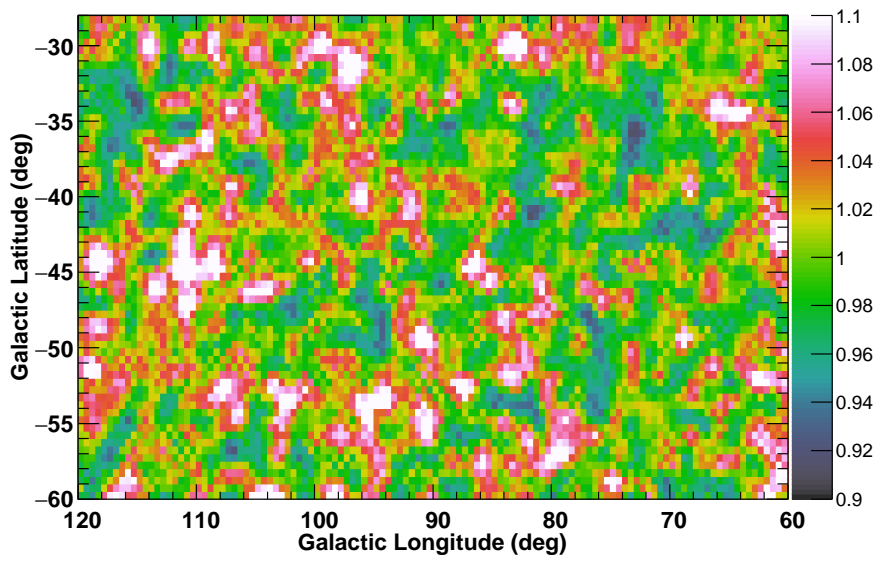

(a)

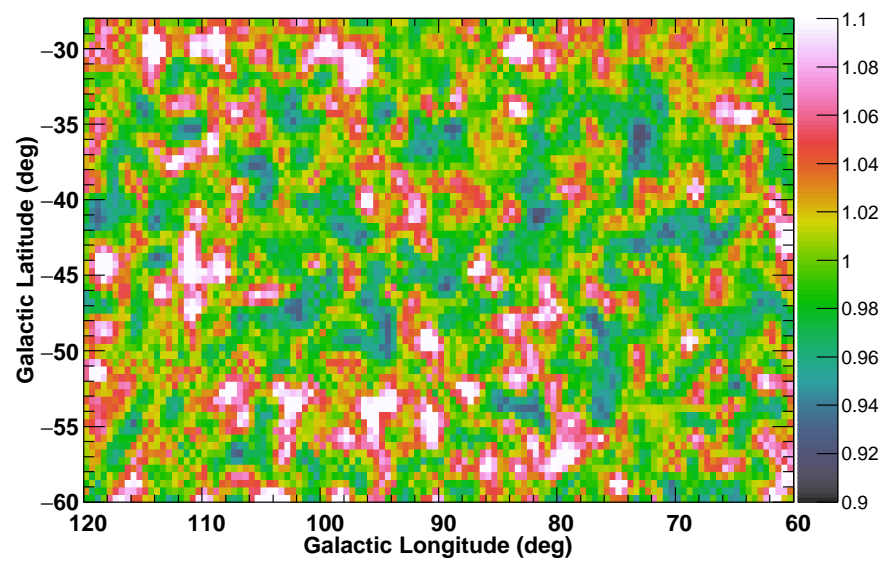

(b)

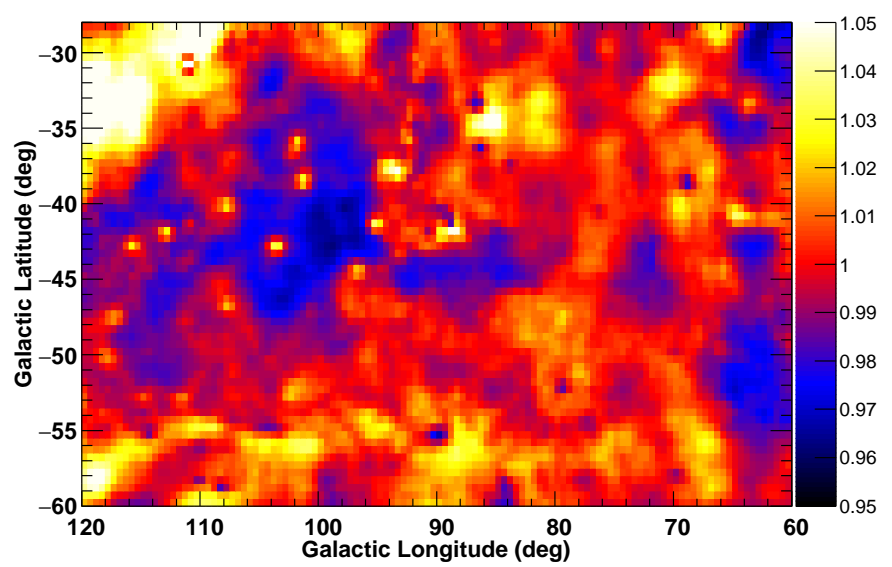

(c)

Figure D5. (a) The data/model ratio map obtained by the conventional template-fitting method. (b) The same map, but obtained by the analysis using the $N\left(\mathrm{H}_{\text {tot,mod }}\right)$ map. (c) The ratio of $\gamma$-ray model maps of the conventional templatefitting method to the analysis using the $N\left(\mathrm{H}_{\text {tot,mod }}\right)$ map. In panels (a) and (b), a smoothing with a k5a kernel (1-2-5-2-1 two-dimensional boxcar smoothing) in ROOT framework (https://root.cern.ch) was applied. Positive/negative spikes in panel (c) correcpond to point sources considered. 


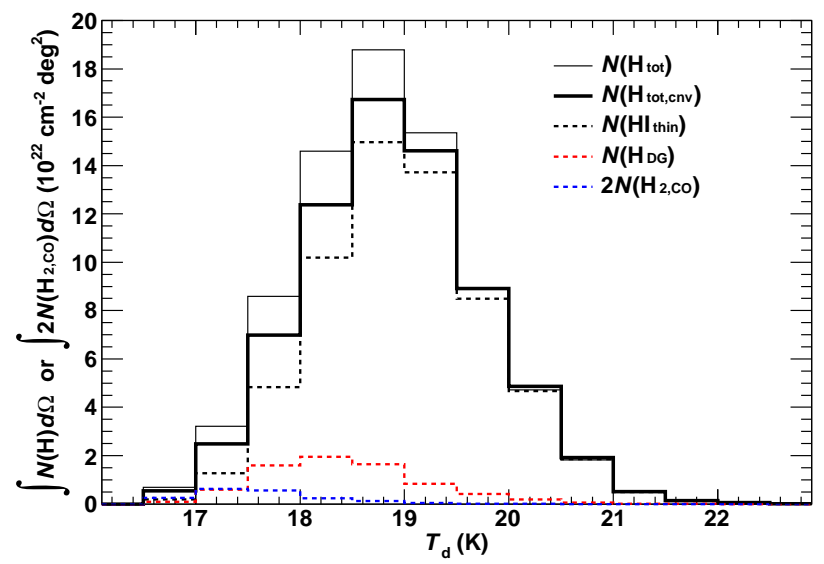

Figure D6. The distributions of the integrated hydrogen column density inferred from $W_{\mathrm{H}}$ for the optically thin case $\left(\int N\left(\mathrm{HI}_{\text {thin }}\right) d \Omega\right)$, that inferred from the dark gas template $\left(\int X_{\mathrm{DG}} R_{\mathrm{res}}^{\mathrm{DG}} d \Omega\right)$, and that inferred from $\mathrm{CO}$ emission ( $\int 2 X_{\mathrm{CO}} W_{\mathrm{CO}} d \Omega$ as a measure of CO-bright $\mathrm{H}_{2}$ ) obtained by the conventional template-fitting method (dotted histograms). The integral of the total gas column density is also shown as the thick solid histogram. For comparison, the integral of the $N\left(\mathrm{H}_{\text {tot,mod }}\right)$ (already shown in Figure $\left.9 \mathrm{~b}\right)$ is also presented as the thin solid histogram. 


\section{REFERENCES}

Abdo, A. A., Ackermann, M., Ajello, M., et al. 2009a, Astropart. Phys., 32, 193

Abdo, A. A., Ackermann, M., Ajello, M., et al. 2009b, ApJ, 703, 1249

Abdo, A. A., Ackermann, M., Ajello, M., et al. 2010, ApJ, 710, 133

Acero, F., Ackermann, M., Ajello, M., et al., 2015, ApJS, 23, 41

Ackermann, M., Ajello, M., Baldini, L., et al. 2011, ApJ, 726, 81

Ackermann, M., Ajello, M., Atwood, W. B., et al. 2012a, ApJ, 750,3

Ackermann, M., Ajello, M., Allafort, A., et al. 2012b, ApJ, 755, 22

Aharonian, F. A., \& Atoyan, A. M. 2000, A\&A, 362, 937

Atwood, W. B., Abdo, A. A., Ackermann, M., et al. 2009, ApJ, 697, 1071

Bennett, C. L., Larson, D., Weiland, J. L., et al. 2013, ApJS, 208, 20

Bohlin, R. C., Savage, B. D., \& Drake, J. F. 1978, ApJ, 224, 132

Clemens, D. P. 1985, ApJ, 295, 422

Casandjian, J.-M. 2015, ApJ, 806, 240

Dame, T. M., Hartmann, D., \& Thaddeus, P. 2001, ApJ, 547, 792

Dame, T. M. 2011, arXiv:1101.1499

Däppen, W. 2000, in Allen's Astrophysical Quantities, ed. A. N. Cox (4th ed.; New York: Springer), 27 de Palma, F., Brandt, T. J., Johannesson, G., \& Tibaldo, L. 2012, arXiv:1304.1395

Dickey, J. M., \& Lockman, F. J. 1990, ARA\&A, 28, 215

Draine, B. T., \& Li, A. 2007, ApJ, 657, 810

Ferriere, K. M. 2001, Rev. Mod. Phys., 73, 1031

Fukui, Y., Okamoto, R., Kaji, R., et al. 2014, ApJ, 796, 59

Fukui, Y., Torii, K., Onishi, T., et al. 2015, ApJ, 798, 6

Górski, K. M., Hivon, E., Banday, A. J., et al. 2005, ApJ, 622, 759
Grenier, I. A., Casandjian, J.-M., \& Terrier, R. 2005, Science, 307, 1292

Grenier, I. A., Black, J. H., \& Strong, A. W. 2015, ARA\&A, 53, 199

Kalberla, P. M. W., Burton, W. B., Hartmann, D., et al. 2005, A\&A, 440, 775

Kiss, Cs., Moór, A., \& Tóth, L. V. 2004, A\&A, 418, 131

Mattox, J. R., Bertsch, D. L., Chiang, J., et al. 1996, ApJ, 461, 396

Mori, K. 2009, Astropart.Phys., 31, 341

Planck Collaboration XXIV 2011, A\&A, 536, 24

Planck Collaboration XI 2014, A\&A, 571, 11

Planck Collaboration XXVIII 2015, A\&A, 582, 31

Porter, T. A., Moskalenko, I. V., Strong, A. W., et al., 2008, ApJ, 682, 400

Reach, W. T., Bon-Chul, K., \& Carl, H. 1994, ApJ, 429, 672

Smith, R. J., Glover, S. C. O., Clark, P., et al., 2014, MNRAS, 441,1628

Strong, A. W., \& Moskalenko, I., 1998, ApJ, 509, 212

Strong, A. W., Moskalenko, I.V., \& Ptuskin, V. S. 2007, ARA\&A, 57, 285

Tibaldo, L., Digel., S. W., Casandjian, J.-M., et al., 2015, ApJ, 807, 161

Wakker, B. P. 2001, ApJS, 136, 463

Welty, D. E, Hobbs, L. M., Penprase, B. E., \& Blitz, L. 1989, ApJ, 346, 232

Wolfire, M. G., Hollenbach, D., \& McKee, C. F. 2010, ApJ, 716, 1191

Yamamoto, H., Onishi, T., Mizuno, A., \& Fukui, Y. 2003, ApJ, 592, 217

Yamamoto, H., Kawamura, A., Tachihara, K., et al. 2006, ApJ, 642, 307

Ysard, N., Köhler, M., Jones, A., et al. 2015, A\&A, 577, 110 